\title{
ARTICLE
}

\section{Inhibition of inflammasome activation by Coxiella burnetii type IV secretion system effector IcaA}

\author{
Larissa D. Cunha ${ }^{1}{ }^{\dagger}$, Juliana M. Ribeiro ${ }^{1}$, Talita D. Fernandes ${ }^{1}$, Liliana M. Massis ${ }^{1}$, Chen Ai Khoo ${ }^{2}$,
} Jennifer H. Moffatt ${ }^{2}$, Hayley J. Newton ${ }^{2}$, Craig R. Roy ${ }^{3} \&$ Dario S. Zamboni ${ }^{1}$

Coxiella burnetii is a highly infectious bacterium that promotes its own replication in macrophages by inhibiting several host cell responses. Here, we show that $C$. burnetii inhibits caspase- 1 activation in primary mouse macrophages. By using co-infection experiments, we determine that the infection of macrophages with $C$. burnetii inhibits the caspase-11-mediated non-canonical activation of the NLRP3 inflammasome induced by subsequent infection with Escherichia coli or Legionella pneumophila. Genetic screening using flagellin mutants of L. pneumophila as a surrogate host, reveals a novel C. burnetii gene (IcaA) involved in the inhibition of caspase activation. Expression of IcaA in L. pneumophila inhibited the caspase-11 activation in macrophages. Moreover, ica $A^{-}$mutants of $C$. burnetii failed to suppress the caspase-11-mediated inflammasome activation induced by $L$. pneumophila. Our data reveal IcaA as a novel C. burnetii effector protein that is secreted by the Dot/Icm type IV secretion system and interferes with the caspase-11-induced, non-canonical activation of the inflammasome.

\footnotetext{
${ }^{1}$ Department of Cell Biology, Medical School of Ribeirão Preto, University of São Paulo (FMRP/USP), Ribeirão Preto, Sao Paulo 14049-900, Brazil.

2 Department of Microbiology and Immunology, University of Melbourne at the Peter Doherty Institute for Infection and Immunity, Melbourne, Victoria 3000, Australia. ${ }^{3}$ Department of Microbial Pathogenesis, Yale University School of Medicine, New Haven, Connecticut 06536, USA. † Present address: Department of Immunology, St. Jude Children's Research Hospital, Memphis, Tennessee 38105, USA. Correspondence and requests for materials should be addressed to D.S.Z. (email: dszamboni@fmrp.usp.br).
} 
nnate immune cells have developed systems to signal and mount appropriate responses to pathogenic microbes by recognizing conserved molecules present in microbes (pathogen-associated molecular patterns, PAMPs) ${ }^{1}$. For intracellular pathogens, cytosolic nucleotide binding domain and leucine rich repeat containing proteins (NLR) play a pivotal role in recognizing and mounting effective immune responses. Caspase- 1 activation mediated by NLR leads to interleukin-1 $\beta$ (IL-1 $\beta$ ), IL-18, IL-1 $\alpha$ release, membrane permeabilization and a specific form of inflammatory cell death called pyroptosis ${ }^{2}$. The activation of caspase- 1 by NLR occurs in a molecular platform known as an inflammasome ${ }^{3}$. Notably, the adaptor protein apoptosis-associated speck-like protein containing a carboxy-terminal CARD (ASC) is involved in the structure of different inflammasomes ${ }^{2}$. Several NLR, including NLRC4, NLRP1, NLRP3, NLRP6, NLRP12, neuronal apoptosis inhibitory protein 2 (NAIP2), NAIP5, NAIP6 and the cytosolic non-NLR receptor absent in melanoma 2 (AIM2), have been shown to activate the inflammasome in response to molecules derived from the different classes of pathogens. In particular, the specialized secretion systems of pathogenic Gram-negative bacteria have been shown to be a direct PAMP for inflammasome activation, and to be involved in processes that lead to delivery of specific PAMPs into the host cell cytoplasm ${ }^{4-8}$. In the case of the intracellular Gram-negative bacterium Legionella pneumophila, caspase-1 activation is readily triggered in response to the recognition of bacterial flagellin by NAIP5 and NLRC4, a process that requires the expression of a functional type IV secretion system (T4SS) called defect in organelle trafficking/ intracellular multiplication $(\mathrm{Dot} / \mathrm{Icm})^{9-11}$. Most recently, caspase-11 has emerged as an important regulator of inflammasome activation in response to the lipopolysaccharide (LPS) of Gram-negative bacterial species that either express a secretion system or escape to the host cell cytosol ${ }^{12-18}$. Caspase11 mediates NLRP3-dependent caspase-1 activation (called the non-canonical activation of the NLRP3 inflammasome), but also induces a caspase-1-independent membrane permeabilization that leads to pyroptosis and the release of inflammatory cytokines, including IL- $1 \alpha$.

Given the presence of a large repertoire of intracellular patternrecognition receptors mediating inflammasome activation and pyroptotic cell death, the bona fide intracellular pathogens have developed efficient strategies to manipulate host cell functions and subvert host surveillance (reviewed in Cunha et al. ${ }^{19}$ ). In this sense, the expression of specialized secretion systems that mediate the injection of pathogen effector proteins directly into host cell cytosol constitutes a widespread strategy used by intracellular bacterial pathogens to manipulate host cell functions ${ }^{20-22}$. For intracellular pathogens that target innate immune cells and are associated with severe and highly threatening chronic diseases, such as Chlamydia trachomatis, Coxiella burnetii and Mycobacterium tuberculosis, it is predictable that these pathogens encode unique defence mechanisms to subvert inflammasome activation. In fact, factors that inhibit inflammasome activation have been identified in M. tuberculosis and in C. trachomatis, although the molecular mechanisms of inflammasome inhibition and host cell targets are unclear ${ }^{23-25}$.

The intracellular bacterial pathogen C. burnetii is the causative agent of an acute pneumonia-like disease called Q fever, which may lead to endocarditis or hepatitis ${ }^{26}$. The bacteria replicate within a phagolysosomal vacuole and infect primarily alveolar macrophages $(\mathrm{AMs})^{27}$. C. burnetii is highly specialized to subvert host cell functions, including the avoidance of TLRs recognition, the inhibition of apoptosis and the modulation of diverse vesicle traffic pathways ${ }^{28-39}$. However, immunocompetent individuals can effectively control bacterial multiplication, thus emphasizing that effective immune responses are critical for host resistance. C. burnetii is evolutionarily close to L. pneumophila, belonging to the order of Legionellales. Importantly, these pathogens share a homologous Dot/Icm type IV secretion system ${ }^{40}$. Historically, the genetic manipulation of $C$. burnetii has not been easily achieved, and as such, L. pneumophila has been used as a surrogate host to characterize and identify the C. burnetii Dot/Icm secretion system $^{41,42}$. C. burnetii encodes diverse putative effector proteins in its genome, some of them with sequence motifs predicted to mediate eukaryotic protein-protein interactions such as ankyrin repeat-containing and leucine-rich repetitioncontaining domains ${ }^{40,43}$. More than 100 putative C. burnetii effectors identified by genetic and bioinformatic whole-genome screens have been shown to be secreted by the Dot/Icm system of L. pneumophila ${ }^{44-50}$. Despite this massive identification of the C. burnetii effectors, the molecular function of very few effector proteins has been defined. Because $C$. burnetii is a highly virulent intracellular pathogen and very few cells ( $<5$ organisms) are sufficient to induce disease in a healthy human being, we investigated whether inflammasome activation occurs in response to $C$. burnetii infection. We found that $C$. burnetii inhibits the non-canonical activation of the NLRP3 inflammasome, which occurs in a process dependent on caspase-11 and independent of NLRC4 and bacterial flagellin. To search for the mechanism underlying the inhibition of the inflammasome by the bacterium, we screened potential effectors using L. pneumophila as a surrogate host and identified a C. burnetii effector protein, denoted herein as IcaA (Inhibition of caspase activation), that interferes with the caspase-11-mediated, non-canonical activation of the inflammasome. C. burnetii mutants for icaA fail to inhibit caspase-11 activation induced by fla $A$ mutants of L. pneumophila. These findings unveil the process of inflammasome inhibition by $C$. burnetii and therefore contribute to our understanding of the molecular mechanisms underlying the virulence strategies used by this highly threatening obligate intracellular pathogen.

\section{Results}

C. burnetii infection does not stimulate inflammasome activation. To investigate whether $C$. burnetii induces caspase- 1 activation, we infected murine bone marrow-derived macrophages (BMDMs) for $24 \mathrm{~h}$ and assessed the processing of caspase- 1 and pro-IL-1 $\beta$ by western blot. We found that the infection of wild-type C57BL/6 BMDMs (BL/6 BMDMs) with C. burnetii does not induce the cleavage of caspase-1 p20 subunit (Fig. 1a). As a control, we used L. pneumophila, which readily triggers the processing of these proteins in infected BMDMs (Fig. 1a). To further evaluate caspase-1 activation in response to C. burnetii, we assessed caspase- 1 activation in BMDMs using the FAM-YVAD-FMK (FLICA), a previously described fluorescent probe that specifically binds the active form of caspase- 1 while inhibiting its autoproteolytic processing ${ }^{11}$. We found that in contrast to L. pneumophila infection, C. burnetii infection does not induce an increase in the percentage of cells stained with FLICA, as measured by flow cytometry (Fig. 1b,c). As a control, we used BMDMs from a Casp $1 / 11^{-}$mouse to indicate that this probe is specific to caspase-1. Finally, we measured the secretion of IL-1 $\beta$ as readout for caspase- 1 activation. We found that as opposed to L. pneumophila infection, C. burnetii infection does not induce the secretion of IL-1 $\beta$ (Fig. 1a,d). Altogether, these data show that $C$. burnetii does not trigger caspase-1 activation in BMDMs.

C. burnetii inhibits NLRP3 activation induced by caspase-11. Although macrophages use multiple sensors to detect 
a

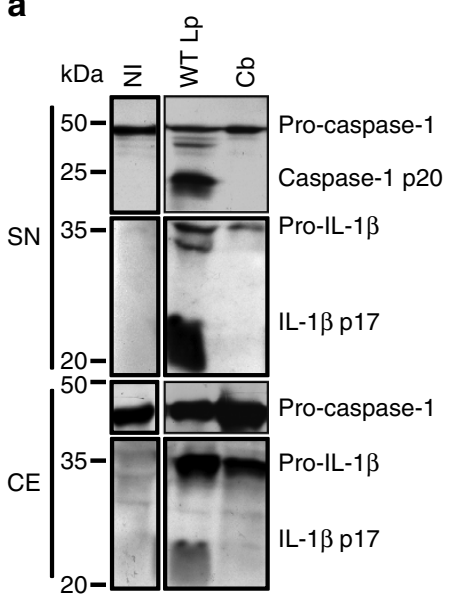

b
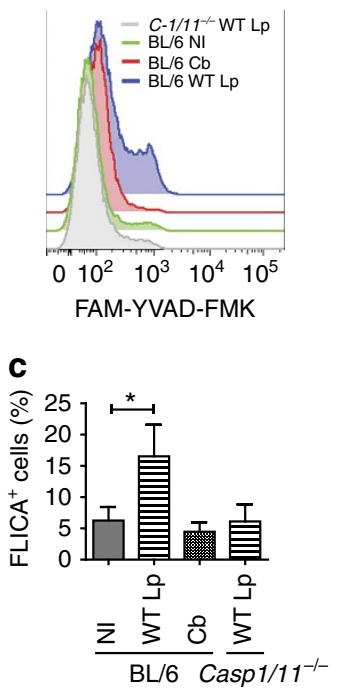

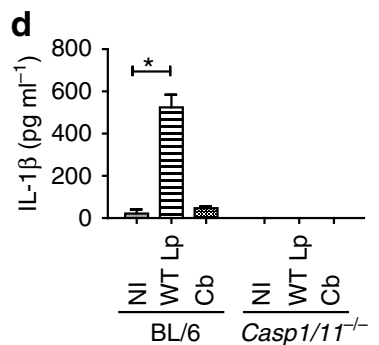

Figure 1 | Caspase-1 is not activated in macrophages infected with C. burnetii. Bone marrow-derived macrophages (BMDMs) remained noninfected (NI) or were infected with wild-type L. pneumophila for $9 \mathrm{~h}$ (WT Lp, multiplicity of infection (MOI) 10) or C. burnetii for 24 h (Cb, MOI 30). (a) Immunoblot showing processed p20 subunit of caspase-1 (caspase-1 p20), unprocessed caspase-1 (pro-caspase-1), p17 subunit of mature IL-1 (IL-1 $\beta$ p17) and pro-IL$1 \beta$, as determined in the supernatant (SN) and in the cell extract (CE). (b) Flow analysis of FLICA staining in BL/6 BMDM noninfected (green curve), infected with Cb (red curve) or infected with WT Lp (blue curve) and Casp1/11-/- BMDMs infected with WT Lp (grey curve). (c) Quantification of FLICApositive cells in (b). (d) IL-1 $\beta$ secretion in infected BMDMs was determined by ELISA. Data in (c,d) are expressed as average \pm s.e.m. of triplicate wells and significance was calculated with $t$-test. ${ }^{\star} P<0.05$ compared with $\mathrm{NI}$ cells. Data are representative of at least three independent experiments. Full blots are presented in the Supplementary Fig. 10.

Gram-negative bacteria, C. burnetii can evade immune responses and interfere with different cellular process to establish a replicative niche in these cells. Therefore, we investigated whether the absence of caspase- 1 activation results from the active process of inhibition by C. burnetii. Previous work has demonstrated that C. burnetii and L. pneumophila establish distinct and functional replicative vacuoles in co-infected $\mathrm{BMDMs}^{51}$. We thus performed co-infection studies to evaluate whether C. burnetii can actively inhibit the inflammasome activation triggered by L. pneumophila. We found that pre-infection of BMDMs with C. burnetii for $24 \mathrm{~h}$ resulted in reduced caspase- 1 activation and pro-IL-1 $\beta$ cleavage in response to a second infection with L. pneumophila (Fig. 2a). Notably, pre-infection with $C$. burnetii induced a significantly higher expression of pro-casp-1 and pro-IL-1 $\beta$, than single infections with $L$. pneumophila, possibly because of the presence of C. burnetii PAMPs during the 24-h pre-stimulation. To guarantee that the cells were stimulated similarly during the co-infection experiments, we pre-infected BMDMs with live or paraformaldehyde (PFA)-fixed C. burnetii before infections with L. pneumophila. We found that PFA-inactivated C. burnetii induced a similar expression of pro-casp-1 as the live bacteria, but only live $C$. burnetii inhibited caspase- 1 activation in response to L. pneumophila (Supplementary Fig. 1a). These data indicate that the inhibition of caspase- 1 activation is an actively induced process that requires $C$. burnetii metabolic activity. Caspase- 1 activation in response to L. pneumophila occurs via at least two distinct pathways, one dependent on and another independent of bacterial flagellin ${ }^{52}$. To evaluate whether C. burnetii inhibits the flagellin-dependent or -independent pathway triggered by L. pneumophila infection, we performed co-infections of $C$. burnetii with wild-type (WT Lp) or flagellin-deficient $\left(\right.$ fla $\left.^{-}\right)$L. pneumophila. We found that C. burnetii pre-infection inhibits caspase- 1 activation and IL-1 $\beta$ processing in response to either WT Lp or flaA ${ }^{-}$L. pneumophila (Fig. 2a). Strikingly, the co-infections performed with flaA $^{-}$ L. pneumophila revealed that the flagellin-independent pathway for caspase- 1 activation was abolished when the BMDMs were pre-infected with C. burnetii (Fig. 2a). These data indicate that C. burnetii effectively inhibits the flagellin-independent pathway for caspase-1 activation. Similar experiments performed with PFA-fixed C. burnetii indicated that live C. burnetii is required for the inhibition of caspase- 1 activation in response to $\mathrm{flaA}^{-}$ L. pneumophila (Supplementary Fig. 1b).

These findings were further confirmed by the assessment of endogenous caspase- 1 activation with the FLICA assay. BMDMs pre-infected with $C$. burnetii showed a reduction in caspase-1 activation in response to infection with both the WT Lp and flaA $^{-}$L. pneumophila (Fig. 2b-d). As demonstrated by western blot, when co-infections were performed using $\mathrm{flaA}^{-}$ L. pneumophila, we found that BMDMs do not trigger caspase-1 activation (Fig. 2c,d). Pre-infections with C. burnetii did not reduce the internalization of $L$. pneumophila in BMDMs (Supplementary Fig. 2), confirming that this phenomenon is not caused by differences in L. pneumophila internalization after a previous exposure to $C$. burnetii. The C. burnetii-mediated inhibition of caspase- 1 activation induced by $L$. pneumophila was also assessed by measuring the secretion of active IL- $1 \beta$ by enzyme-linked immunosorbent assay (ELISA). We found that pre-infection with live C. burnetii inhibited the secretion of IL-1 $\beta$ in response to infection with WT Lp, and abolished the secretion of IL-1 $\beta$ in response to infection with flaA $^{-}$L. pneumophila (Fig. 2e).

We investigated whether C. burnetii is able to inhibit caspase-1 activation in response to other stimuli. Thus, BMDMs pre-infected or not with C. burnetii were stimulated with LPS + ATP or with LPS + dsDNA to trigger the activation of the inflammasomes dependent on NLRP3 and AIM2, respectively. We found that $C$. burnetii fails to inhibit caspase-1 activation in response to ATP (Supplementary Fig. 3a,c) and dsDNA (Supplementary Fig. 3b,d). These results argue that $C$. burnetii does not directly inhibit caspase- 1 activation but may interfere with upstream events in the signalling cascade of the inflammasome 
a

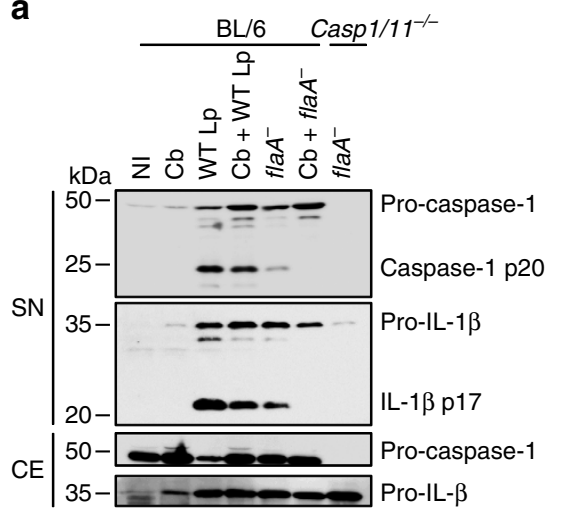

b

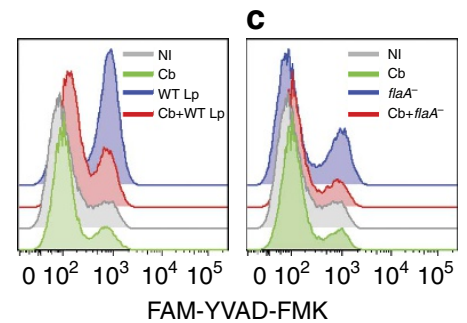

d

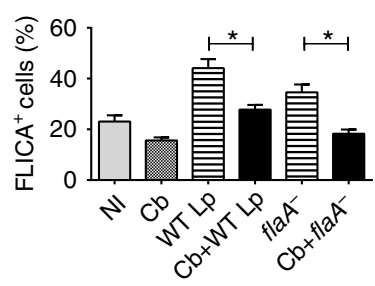

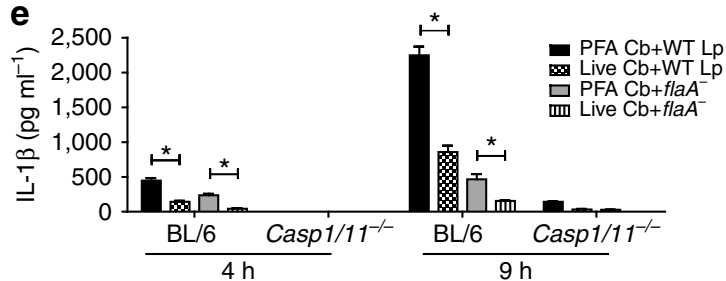

f

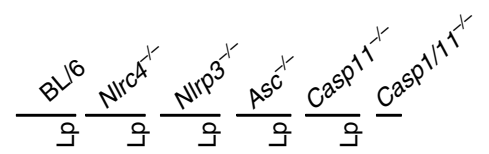

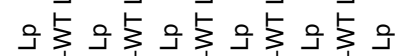

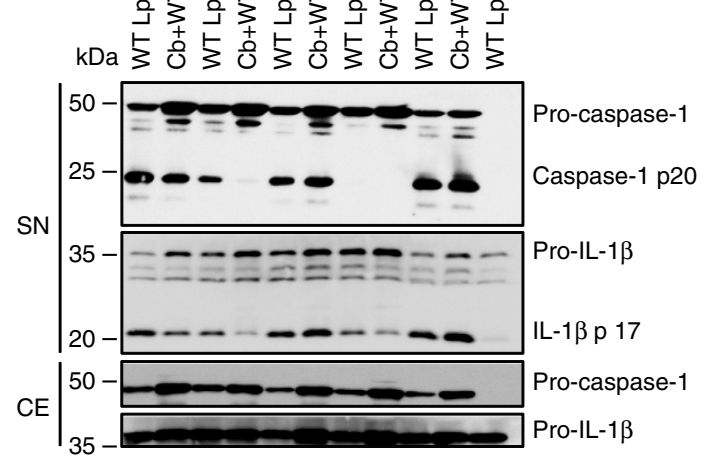

Figure 2 | C. burnetii inhibits the caspase-11-mediated non-canonical activation of the NLRP3 inflammasome induced by L. pneumophila. Bone marrowderived macrophages (BMDMs) were left noninfected $(\mathrm{NI})$ or were infected with $\mathrm{C}$. burnetii $(\mathrm{Cb}, \mathrm{MOI} 30)$ for $24 \mathrm{~h}$ and further infected with wild-type L. pneumophila (WT Lp, MOI 10) or flaA- (MOI 10) for $9 \mathrm{~h}$. Co-infected BMDMs are indicated (Cb + WT Lp or $\mathrm{Cb}+$ fla $A^{-}$). (a) Immunoblot showing levels of processed p20 subunit of caspase-1 (caspase-1 p20), unprocessed caspase-1 (pro-caspase-1), p17 subunit of mature IL-1 3 (IL-1 $\beta$ p17) and pro-IL$1 \beta$, as determined in supernatant (SN) and cell extract (CE). (b,c) Flow analysis of FLICA staining in BL/6 BMDM noninfected (grey curve), infected with $\mathrm{Cb}$ (green curve), infected with L. pneumophila (blue curve) or co-infected (red curve). (d) Quantification of FLICA-positive cells in (b,c). (e) IL-1 $\beta$ secretion in infected BMDMs was determined by ELISA. BMDMs infected with live $\mathrm{C}$. burnetii (live $\mathrm{Cb}$ ) or with PFA-fixed were used. (f) Caspase-1 cleavage and IL-1 $\beta$ secretion were evaluated by immunoblot as in (a). Data in $(\mathbf{d}, \mathbf{e})$ are expressed as average \pm s.e.m. of triplicate wells and significance was calculated with ANOVA. ${ }^{\star} P<0.05$. Data are representative of three $(\mathbf{a}, \mathbf{f})$ and two $(\mathbf{b}-\mathbf{e})$ independent experiments.

triggered by $L$. pneumophila. It was recently demonstrated that $L$. pneumophila triggers the non-canonical activation of the NLRP3 inflammasome in a process independent of flagellin and dependent on caspase-11 (ref. 13). Thus, we used BMDMs from $\mathrm{Nlrc}^{-/-}$, Nlrp3 $3^{-/-}, A s c^{-/-}$or Casp $11^{-/-}$mice to evaluate the requirement of these proteins for the $C$. burnetii-mediated inhibition of caspase-1 activation in response to L. pneumophila. We found that the inhibition of caspase-1 processing occurs in Nlrc4 ${ }^{-/}$BMDMs, a feature that is consistent with the findings that $C$. burnetii inhibits the flagellin-independent pathway for caspase- 1 activation (Fig. 2f). In the absence of ASC, we detected no caspase-1 processing. In Nlrp3 $3^{-/-}$and Casp $11^{-/-}$BMDMs, we found that $C$. burnetii fails to inhibit caspase-1 cleavage and the processing of pro-IL-1 $\beta$ (Fig. $2 \mathrm{f}$ ). This feature is consistent with the hypothesis that $C$. burnetii inhibits the non-canonical activation of the NLRP3 inflammasome, which occurs through caspase-11 and culminates in the activation of the NLRP3/ASC/caspase-1 platform, leading to caspase- 1 cleavage and IL- $1 \beta$ production ${ }^{53}$. It was previously demonstrated that Gram-negative bacteria, such as $E$. coli, or the combination of LPS plus the B subunit of cholera toxin
(CTB) triggers the non-canonical activation of the inflammasome ${ }^{18,53}$. Thus, to test whether C. burnetii inhibits the non-canonical activation of the inflammasome, we pre-infected BMDMs with C. burnetii, stimulated with either LPS + CTB or E. coli and measured caspase- 1 activation. We found that $C$. burnetii pre-infection severely inhibited caspase- 1 activation and pro-IL- $1 \beta$ processing in response to $E$. coli (Fig. 3a). According to previous reports, the $E$. coli-induced activation of caspase- 1 and pro-IL-1 $\beta$ cleavage was dependent on NLRP3 and ASC (Fig. 3a). Supporting the inhibitory activity of $C$. burnetii in the non-canonical inflammasome activation, we found that the release of IL-1 $\beta$ and IL- $1 \alpha$ in response to $E$. coli or LPS + CTB is reduced in BMDMs pre-infected with C. burnetii (Fig. 3b-e). Next, we used a pore formation assay that reflects a caspase-11 activity that operates independently of NLRC4 inflammasome and upstream of the noncanonical activation of the NLRP3 inflammasome ${ }^{13,54}$. By coinfecting cells with $C$. burnetii and $E$. coli, we found that $C$. burnetii is able to inhibit the caspase-11-mediated pore formation induced by E. coli (Fig. 3f-h). In this experiment, we also included BMDMs from a mouse deficient in caspase-1. This mouse, herein called 
a
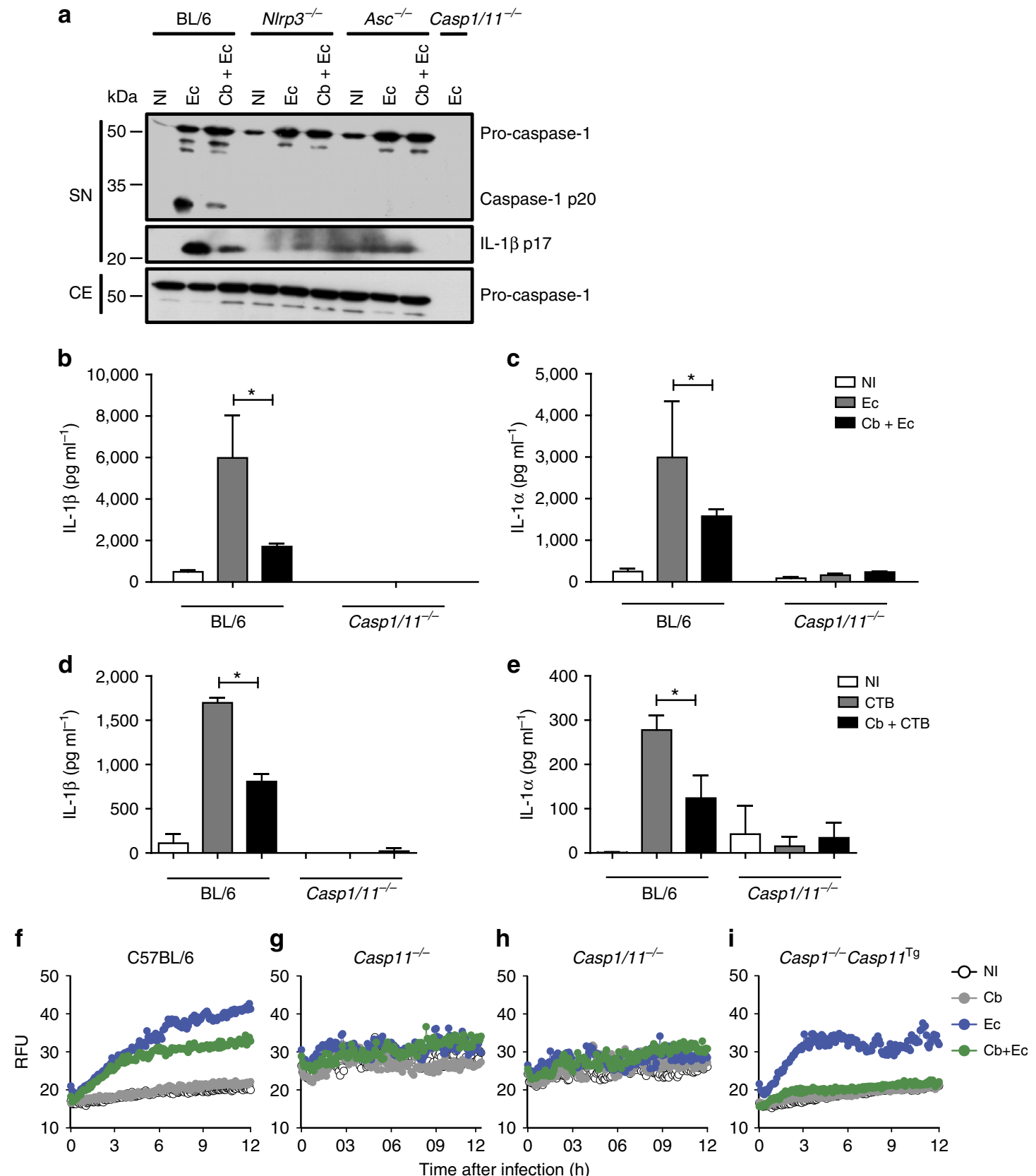

Figure 3 | C. burnetii inhibits caspase-11-dependent pore formation and NLRP3 activation in response to $E$. coli and cholera toxin B. Bone marrowderived macrophages (BMDMs) generated from wild-type C57BL/6 (BL/6), N/rp3 ${ }^{-/-}$, Asc ${ }^{-/-}$or Casp1/11 ${ }^{-/-}$mice were left noninfected (NI) or were infected with C. burnetii (Cb, MOI 30) for $24 \mathrm{~h}$, stimulated with LPS $\left(0.5 \mu \mathrm{g} \mathrm{ml} \mathrm{l}^{-1}\right)$ for $4 \mathrm{~h}$ and infected with E. coli at $\mathrm{MOI} 20$ (Ec or $\left.\mathrm{Cb}+\mathrm{Ec}\right)$ or treated with CTB at $20 \mu \mathrm{g} \mathrm{ml}^{-1}$ (CTB or $\mathrm{Cb}+\mathrm{CTB}$ ). (a) Immunoblot showing levels of processed p20 subunit of caspase-1 (caspase-1 p20), unprocessed caspase-1

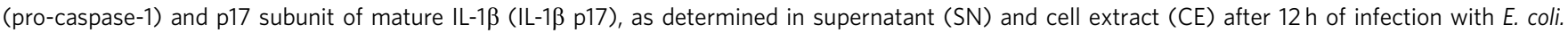
(b) IL-1 $\beta$ and (c) IL- $1 \alpha$ were determined by ELISA from cell supernatants $16 \mathrm{~h}$ after E. coli infection. (d) IL-1 $\beta$ and (e) IL- $1 \alpha$ were determined by ELISA from cell supernatants $16 \mathrm{~h}$ after CTB stimulation. ( $\mathbf{f}-\mathbf{i})$ Pore formation was assessed fluorometrically in real time by the uptake of propidium iodide (RFUs, relative fluorescence units). (b-e) Data are expressed as the average \pm s.e.m. of triplicate wells and significance was calculated with $A N O V A$. ${ }^{\star} P<0.05$. (a-i) Data are representative of two independent experiments.

Casp $1^{-/-} / \operatorname{Casp} 11^{T g}$, is the Casp $1 / 11^{-/-}$expressing a transgene encoding a functional copy of the caspase-11 allele, as described previously ${ }^{53}$. By using Casp1-1-/Casp11 $1^{T g}$ BMDMs, we confirmed that C. burnetii is able to inhibit caspase-11-mediated pore formation induced by $E$. coli in the absence of caspase- 1 (Fig. 3i). Next, we tested whether the inhibition of caspase-11 also operates in primary mouse AMs, which are permissive for $C$. burnetii replication, and are more similar to the relevant cells that harbour C. burnetii during acute $\mathrm{Q}$ fever
(Supplementary Fig. 4a). By using AMs, we found that co-infections with live C. burnetii but not with heat-killed bacteria inhibit the caspase-11-dependent pore formation induced by E. coli in primary AMs similarly to BMDMs (Supplementary Fig. 4b,c).

A genetic screen using $L$. pneumophila identifies $C$. burnetii IcaA. We used L. pneumophila as a surrogate host to screen the specific C. burnetii effector proteins involved in the inhibition of 
non-canonical inflammasome activation, and we constructed a library of $\mathrm{flaA}^{-}$mutants of L. pneumophila expressing C. burnetii effectors that have been confirmed to be secreted via the L. pneumophila type IV secretion system ${ }^{44,45}$. We obtained L. pneumophila clones with a confirmed expression of $24 \mathrm{C}$. burnetii effectors (Supplementary Fig. 5a,b). L. pneumophila mutants expressing $C$. burnetii genes were screened by western blot for the induction of caspase-1 cleavage in infected BMDMs. This screening identified a strain that induced neither caspase-1 activation nor IL-1 $\beta$ maturation, which was the clone of L. pneumophila that produces the effector protein annotated as CBU1823 (Supplementary Fig. 5c-e). Although biochemical activities displayed by the CBU1823 protein are yet unknown, this protein has been shown to be secreted into host cells through the L. pneumophila Dot/Icm system ${ }^{44,45}$. In addition, a recent report demonstrated that CBU1823 is translocated into BMDMs during $C$. burnetii infection in a process dependent on the C. burnetii Dot/Icm system ${ }^{55}$. Because we identified CBU1823 as a C. burnetii Dot/Icm effector protein that inhibits caspase activation, we named this protein IcaA. IcaA shows no known protein domain and has no significant homology to any other eukaryotic or bacterial protein to hint at its mechanisms of function.

We further tested whether the expression of IcaA in different strains of $\mathrm{flaA}^{-}$L. pneumophila displayed reduced caspase-1 cleavage in BMDMs. We found that the inhibition of caspase-1 cleavage by IcaA occurred in both JR32 and Lp01 backgrounds (Supplementary Fig. 6a). Inhibition also occurred when we used the strain Lp02, which is a thymidine auxotroph and does not replicate intracellularly in the absence of thymidine supplementation (Supplementary Fig. 6a). Hence, IcaA-mediated caspase-1 inhibition is independent of bacterial replication. Next, we investigated whether the expression of IcaA influenced L. pneumophila fitness. We compared $\mathrm{flaA}^{-}$mutants of L. pneumophila expressing IcaA $\left(f l a A^{-} / \mathrm{pIcaA}\right)$ with those encoding the empty vector $\left(f l a A^{-} / \mathrm{pVec}\right)$ in assays that measured replication in axenic media, internalization in BMDMs and the induction of cytokines. We found that the expression of IcaA does not affect the bacterial replication in liquid ACES-buffered yeast extract (AYE) axenic media, bacterial internalization in BMDMs or the induction of IL-12 in BMDMs (Supplementary Fig. 6b-d). Moreover, the expression of IcaA or the unrelated $C$. burnetii effector protein AnkH in $f l a A^{-}$L. pneumophila did not interfere with internalization or the early bacterial replication in BMDMs at $9 \mathrm{~h}$ of infection compared with the bacteria encoding the empty vector (Supplementary Fig. 6e,f). The bacteria encoding pIcaA, but not those encoding the $\mathrm{pVec}$, showed a reduced activation of caspase-1 and secretion of IL-1 $\beta$ (Supplementary Fig. 6a,g).

Our studies, using pre-infections with live C. burnetii, supported the findings that $C$. burnetii inhibits the non-canonical activation of the inflammasome. Therefore, we used the $\mathrm{flaA}^{-}$ L. pneumophila-carrying pIcaA (or pVec) to evaluate whether IcaA is involved in the inhibition of the caspase-11-mediated, non-canonical activation of the NLRP3 inflammasome. Initially, we investigated whether caspase-11 is required for the IcaA-mediated inhibition of caspase- 1 activation. We found that whereas $\mathrm{flaA}^{-}$mutants encoding $\mathrm{pVec}$ trigger caspase-1 activation in $\mathrm{BL} / 6 \mathrm{BMDMs}$, the mutants expressing IcaA fail to do so. Importantly, the IcaA-mediated inhibition of caspase-1 activation is not observed in BMDMs from mice of a $129 \mathrm{~S} 6 / \mathrm{SvEv}$ genetic background (herein called 129), a strain that fails to express functional caspase-11 (Fig. 4a,b). To certify that the lack of caspase-1 inhibition in 129 mouse strains was due to the absence of caspase-11, we transduced 129 BMDMs with a retrovirus-encoding caspase-11 (or GFP as a control). The transduced cells expressed a significant amount of p43 and p38 isoforms of pro-caspase-11 (Fig. 4b). By infecting the transduced cells, we found that $\mathrm{flaA}^{-}$L. pneumophila expressing pIcaA inhibits the caspase-1 cleavage in 129 BMDMs expressing caspase-11 but not in cells transduced with retrovirus-encoding GFP (Fig. 4c). In agreement with these findings, we found that $\mathrm{flaA}^{-} / \mathrm{pIcaA}$ mutants do not inhibit caspase-1 cleavage or IL- $1 \beta$ secretion in LPS-primed Casp11 1 - BMDMs in the BL/6 genetic background (Fig. 4d,e). In contrast, in the BL/6 BMDMs, IcaA expression partially inhibited caspase- 1 activation and IL- $1 \beta$ secretion induced by $\mathrm{flaA}^{-}$L. pneumophila, which are convenient proxy measures for caspase-11 activation (Fig. 4d,e). Supporting the role of caspase-11 in the flagellin-independent pathway for caspase-1 activation, we found that BMDMs from Casp11 $1 /$ mice show a reduced activation of caspase-1 and secretion of IL-1 $\beta$ compared with BL/6 BMDMs (Fig. 4d,e).

IcaA inhibits caspase-11-mediated inflammasome activation. Our data reported thus far indicate that IcaA is involved in the inhibition of a caspase-11-dependent, non-canonical activation of inflammasomes. Therefore, we tested whether IcaA interferes with a caspase-11-dependent process that is upstream of caspase1 . We have previously reported that caspase- 11 is involved in the formation of pores in the macrophage membrane that are independent of NLRP3, ASC and caspase-1 (ref. 13). Therefore, we investigated whether $\mathrm{flaA}^{-}$mutants expressing IcaA interfere with caspase-11-mediated pore formation in BMDMs. By analysing the uptake of cell impermeable dye ethidium bromide, we found a partial reduction in pore formation in BMDMs infected with $\mathrm{flaA}^{-} / \mathrm{pIcaA}$ bacteria in comparison to those infected with $\mathrm{flaA}^{-} / \mathrm{pVec}$ (Fig. 5a,b). Next, we performed analyses using the less toxic dye propidium iodide (PI) to evaluate the kinetics of pore formation in real time. By using this approach, we confirmed that IcaA, but not the unrelated $C$. burnetii effector AnkH, partially inhibits the caspase-11-dependent pore formation observed in response to $\mathrm{flaA}^{-}$L. pneumophila (Fig. 5c). Pore formation was not detected in Casp11 $11^{-/-}$ or Casp1/11-/- BMDMs, emphasizing that the flagellinindependent pore formation is caspase-11-dependent (Fig. 5d,e). Notably, the IcaA-mediated inhibition of pore formation occurred normally in BMDMs from Nlrp3 $3^{-/}, A s c^{-/-}$and Casp1 ${ }^{-/-} /$Casp11 ${ }^{T g}$ mice (Fig. 5f-h). These data further confirm that IcaA interferes with caspase-11-mediated responses but not in the downstream components of the NLRP3 inflammasome, such as NLRP3, ASC and caspase-1. To further evaluate whether the IcaA-mediated inhibition of pore formation is specific to caspase-11, we expressed IcaA in flagellated L. pneumophila, which is known to trigger pore formation by a process that is Nlrc4- and caspase-1-dependent but caspase-11independent ${ }^{10,13,52,54,56,57}$. By infecting BMDMs from BL/6, Casp $11^{-/-}$and Casp $1 / 11^{-/-}$mice with $\mathrm{flaA}^{+} / \mathrm{pIcaA}$ or $\mathrm{flaA}^{+} / \mathrm{pVec}$, we found that the IcaA is not effective in inhibiting the flagellin-mediated pore formation that is dependent on caspase-1 but independent of caspase-11 (Supplementary Fig. 7a). As expected, infections performed with $\mathrm{flaA}^{-} / \mathrm{pIcaA}$ inhibited caspase-11-dependent pore formation compared with $\mathrm{flaA}^{-} / \mathrm{pVec}$ (Supplementary Fig. 7b). All these data indicate that IcaA is specifically involved in the inhibition of caspase-11-mediated processes, but it does not influence caspase-1-mediated processes that do not require caspase-11. Our assertion that C. burnetii inhibits a caspase-11dependent pathway implies that the bacteria express molecules that trigger this pathway. To evaluate whether $C$. burnetii is able to trigger caspase-11 activation, we extracted C. burnetii LPS and tested for the induction of pore formation upon 
a

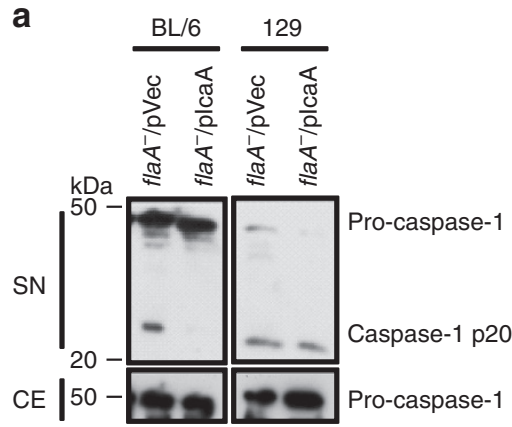

C

C 129 (pGFP) 129 (pCasp11)

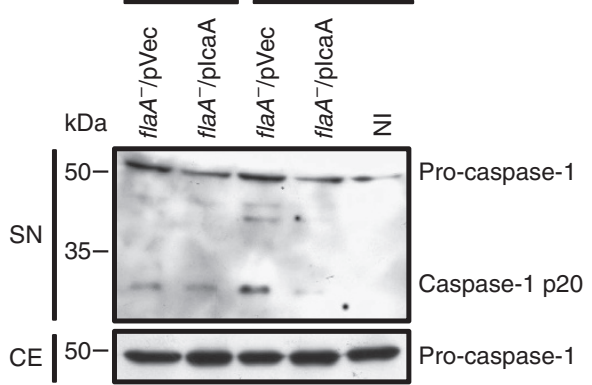

b

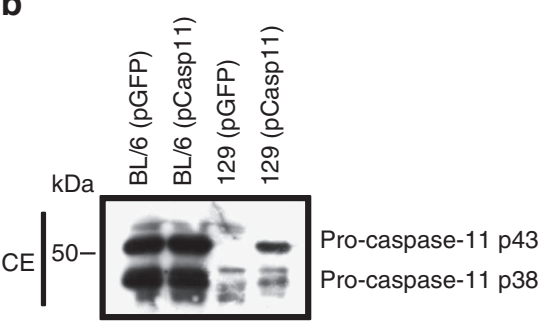

d

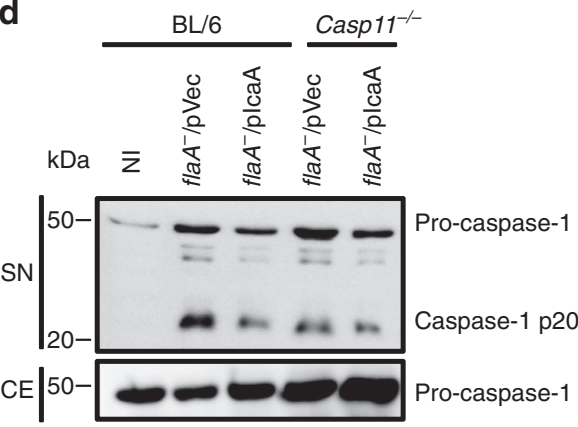

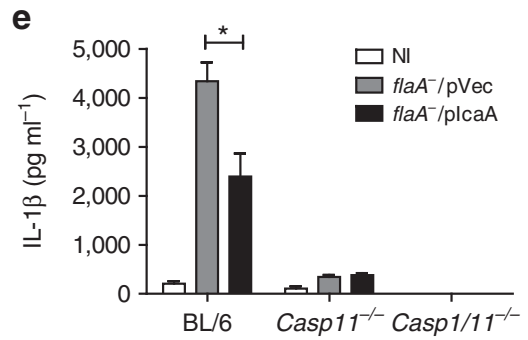

Figure 4 | IcaA requires a functional caspase-11 to inhibit caspase-1 activation. Bone marrow-derived macrophages (BMDMs) generated from C57BL/6 $(\mathrm{BL} / 6)$ and 129S6/SvEv (129) mice were left noninfected (NI) or were infected with flaA ${ }^{-}$mutants of $L$. pneumophila that were transformed either with an empty vector ( $f l a A^{-} / \mathrm{pVec}$ ) or the vector encoding IcaA ( fla $\mathrm{A}^{-} / \mathrm{plcaA}$ ) at $\mathrm{MOI} 10$ for $9 \mathrm{~h}$. (a) Immunoblot showing processed p20 subunit of caspase-1 (caspase-1 p20) and unprocessed caspase-1 (pro-caspase-1), as determined in supernatant (SN) and cell extract (CE). (b) Immunoblot showing the expression of unprocessed isoforms of pro-caspase-11 ( $\mathrm{p} 43$ and $\mathrm{p38}$ ) in uninfected BL/6 and caspase-11-deficient BMDMs derived from 129 mouse strain were transduced with the pMSCV virus encoding GFP (pGFP) or pMSCV encoding full-length caspase-11 (pCasp-11). The expression of unprocessed isoforms of pro-caspase-11 (p43 and p38) is shown by western blot. (c) Immunoblot showing caspase-1 p20 and pro-caspase-1 as determined in SN and CE from transduced BMDMs infected for $9 \mathrm{~h}$. (d) Immunoblot showing caspase-1 p20 and pro-caspase-1 in SN and CE of BMDMs primed with LPS $\left(0.5 \mu \mathrm{g} \mathrm{ml}^{-1}\right)$ and infected for $3 \mathrm{~h}$. (e) IL-1 3 was determined by ELISA from SN of BMDMs primed with LPS for $3 \mathrm{~h}$. Data are expressed as the average \pm s.e.m. of triplicate wells and significance was calculated with ANOVA. ${ }^{\star} P<0.05$. Data are representative of at least two $(\mathbf{b}, \mathbf{c})$ or three $(\mathbf{a}, \mathbf{d}, \mathbf{e})$ independent experiments.

the transfection of BMDMs. We detected a robust pore formation in BMDMs transfected with C. burnetii LPS, which was reduced in Casp1 $1^{-/-} /$Casp $11^{T g}$ and Casp11-/- BMDMs (Supplementary Fig. 8). These data support the hypothesis that C. burnetii expresses molecules that trigger pore formation if delivered in the macrophage cytoplasm, a feature that supports the development of effectors, such as IcaA, to inhibit this response.

Our data generated thus far indicate that IcaA is an effector protein encoded by $C$. burnetii that mediates the inhibition of caspase-11 functions such as pore formation, but it is not clear whether IcaA inhibits caspase-11 activation. Thus, we tested whether IcaA-expressing flaA ${ }^{-}$L. pneumophila can reduce the activation of caspase-11 in the infected BMDMs. To measure caspase-11 activation directly, we pulled down active caspase-11 from macrophages lysates using biotin-VAD-FMK. Active caspase-11-bound to biotin-VAD was concentrated with streptavidin, and the samples were blotted with anti-caspase-11.
We detected a robust caspase-11 activation when BL/6 BMDMs were infected with $\mathrm{flaA}^{-}$L. pneumophila (Fig. 5i). Notably, IcaA expression in $L$. pneumophila reduced caspase-11 activation compared with the bacteria expressing the empty vector (Fig. 5i). The IcaA-mediated inhibition of caspase-11 activation was also observed in experiments performed with macrophages from the Casp1 $1^{-/-} /$Casp11 Tg mice (Fig. 5i). Notably, the overall expression of caspase-11 in these cells is lower than that of C57BL/6 macrophages, possibly because only one copy of the Casp11 $1^{\text {tg }}$ is present in the genome. Regardless of the reduced expression of caspase-11 in the Casp $1^{-/-} /$Casp $11^{T g}$ BMDMs, these data further confirm our assertion that IcaA is involved in the inhibition of caspase-11 activation despite the presence of caspase-1 (Fig. 5e).

icaA $A^{-}$C. burnetii fails to inhibit inflammasome activation. To further evaluate whether the endogenously expressed IcaA in 
a

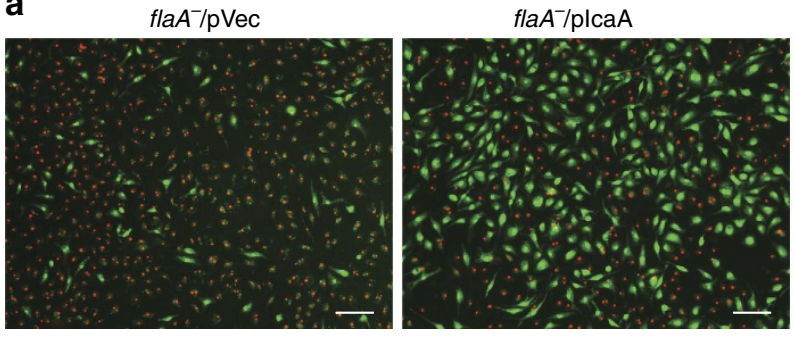

$\mathrm{BL} / 6$

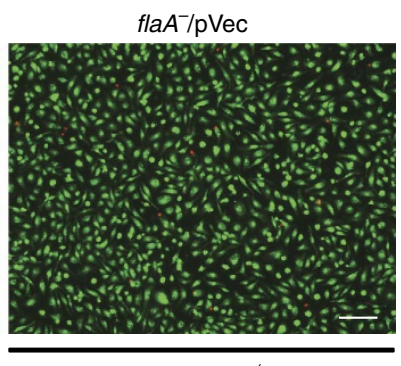

Casp $1 / 11^{-1-}$

b

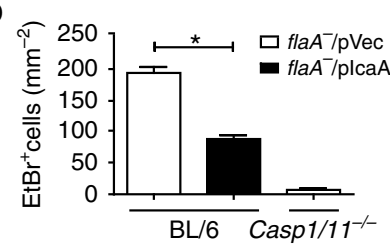

C

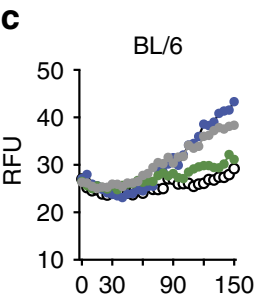

d $\operatorname{Casp11^{-1}}$
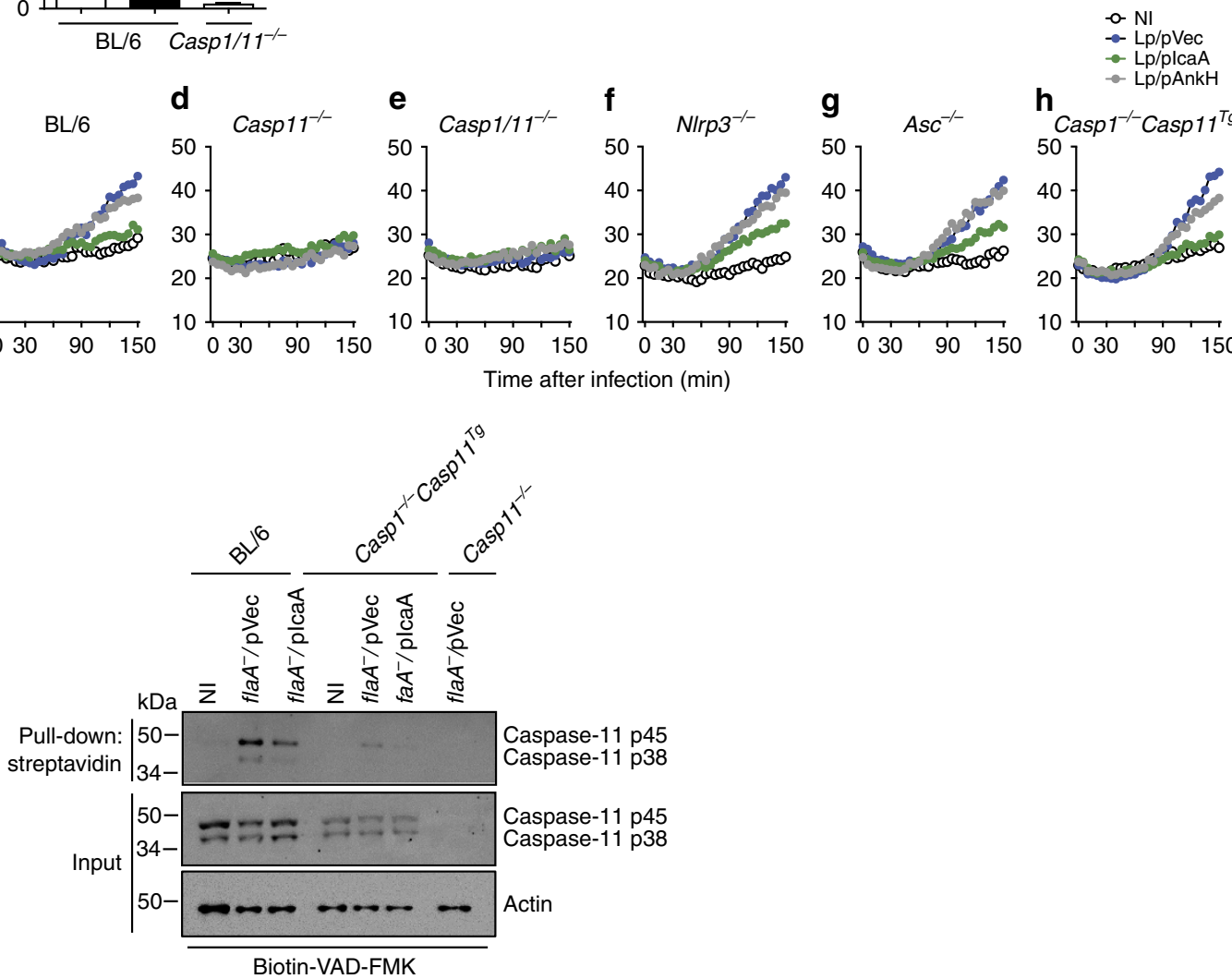

Figure 5 | IcaA does not inhibit caspase-11 activation directly but inhibits a caspase-11 activation upstream of the non-canonical activation of the NLRP3 inflammasome. (a-i) Bone marrow-derived macrophages (BMDMs) were primed with LPS $\left(0.5 \mu \mathrm{g} \mathrm{ml}{ }^{-1}\right)$ for $3 \mathrm{~h}$, remained noninfected $(\mathrm{NI})$ or were infected with flaA $A^{-}$L. pneumophila transformed either with an empty vector (fla $A^{-} / \mathrm{pVec}$ ), the vector encoding IcaA (flaA $-/ \mathrm{plcaA}$ ), or the vector encoding AnkH (fla $A^{-} / \mathrm{pAnkH}$ ) at $\mathrm{MOI}$ 10. (a) Fluorescence micrographs revealing cell permeability, as assessed by quantification of ethidium bromide influx (red). Healthy cells are stained with acridine orange (green). Scale bar, $75 \mu \mathrm{m}$. (b) Quantification of the experiment shown in (a). Data are expressed as the average \pm s.e.m. of triplicate wells and significance was calculated with $t$-test. ${ }^{\star} P<0.05$. (c-h) Pore formation was assessed fluorometrically in real time by the uptake of propidium iodide (RFUs, relative fluorescence units). (i) Immunoblot showing the presence of caspase-11 p45 and p38 in the total cell lysate (input) and pull-down fraction using the agarose-streptavidin fraction of BMDMs treated with biotin-VAD-FMK 15 min before infection. Actin ( $\beta$-actin) was used as a loading control. Data are representative of three (a-h) and two (i) independent experiments.

C. burnetii is involved in the inhibition of the inflammasome activation, we generated the $i c a A^{-}$mutant $C$. burnetii (Supplementary Fig. 9). Initially, we tested whether $i c a A^{-}$is able to trigger caspase-11-dependent responses in single macrophage infections. Measuring caspase-11-dependent pore formation in cells infected with $C$. burnetii, we found that neither the wild-type C. burnetii nor the $i c a A^{-}$mutants triggered a caspase-11dependent pore formation (Fig. 6a). Accordingly, ica $A^{-}$mutants failed to trigger the caspase-11-mediated non-canonical activation of the NLRP3 inflammasome, as measured by the production of IL-1 $\beta$ (Fig. 6b). These data suggest that additional $C$. burnetii processes may be involved in the activities related to the inhibition of inflammasome activation by this bacterium. Next, we tested whether IcaA expression is required for the inhibition of non-canonical inflammasome activation in co-infection experiments. In these experiments, $\mathrm{Nlrc} 4^{-/-} \mathrm{BMDMs}$ or $\mathrm{flaA}^{-}$ 
a

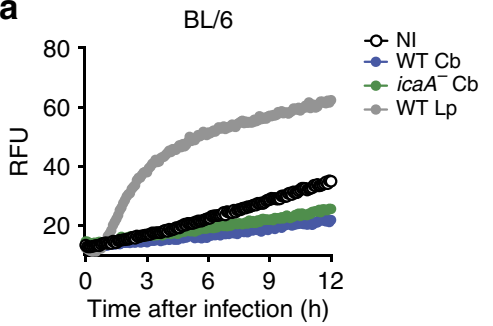

C

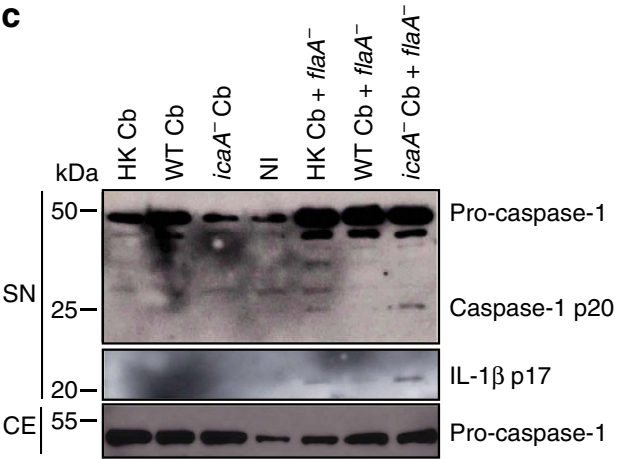

b

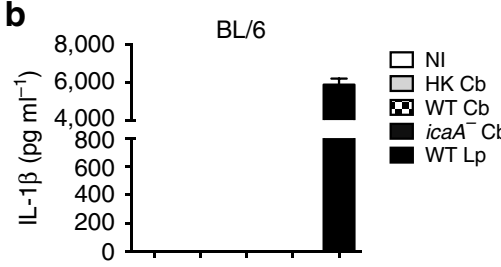

d

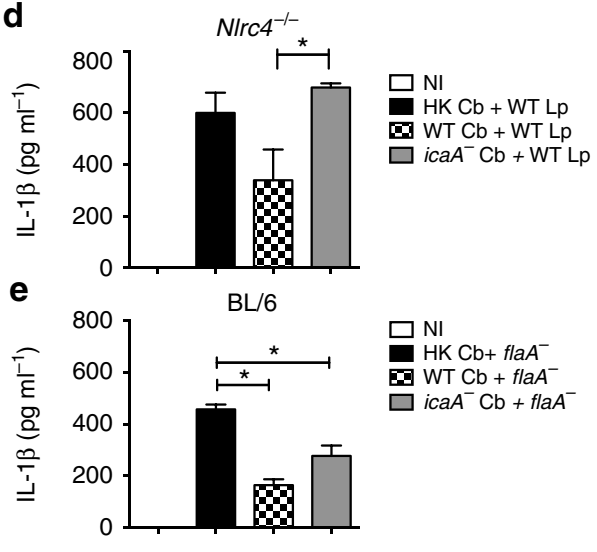

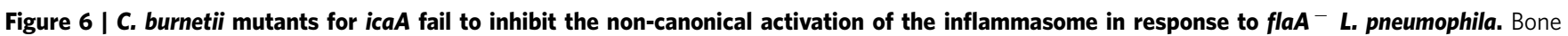
marrow-derived macrophages (BMDMs) were left noninfected ( $\mathrm{NI}$ ) or were infected with heat-killed wild-type ( $\mathrm{HK} \mathrm{Cb}$ ) wild-type (WT Cb) or icaA- mutant (icaA- $\mathrm{Cb}$ ) of $\mathrm{C}$. burnetii for $24 \mathrm{~h}$ at $\mathrm{MOI} 30$, and further infected with wild-type L. pneumophila (WT Lp, MOI 10) or flaA ${ }^{-}$( $\mathrm{MOI}_{10}$ ) for $9 \mathrm{~h}$. Co-infected BMDMs are indicated $\left(\mathrm{Cb}+\mathrm{Lp}\right.$ or $\left.\mathrm{Cb}+\mathrm{flaA}^{-}\right)$. (a) Pore formation was assessed fluorometrically in real time by the uptake of propidium iodide (RFUs, relative fluorescence units). (b,d,e) IL-1 $\beta$ secretion in infected BMDMs was determined by ELISA. Data are expressed as the average \pm s.e.m. of triplicate wells and significance was calculated with $t$-test. ${ }^{\star} P<0.05$. (c) Immunoblot showing processed p20 subunit of caspase-1 (caspase-1 p20), unprocessed caspase-1 (pro-caspase-1) and p17 subunit of mature IL-1 (IL-1 $\beta$ p17), as determined in supernatant (SN) and cell extract (CE). (a-e) Data are representative of at least two independent experiments.

L. pneumophila were used to bypass the activation of NLRC4 inflammasome. We found that, whereas a single infection with wild-type $C$. burnetii or with the $i c a A^{-}$mutants did not trigger caspase-1 activation in BMDMs, the co-infection with icaA ${ }^{-}$ C. burnetii failed to suppress the caspase-1 activation induced by flaA $^{-}$L. pneumophila (Fig. 6c). Accordingly, co-infections performed with wild-type $C$. burnetii, but not with icaA mutants, inhibited the caspase-11-mediated non-canonical activation of the inflammasome, as measured by IL-1 $\beta$ production (Fig. $6 \mathrm{~d}, \mathrm{e}$ ). These data further confirm that endogenously expressed IcaA in C. burnetii is important for the activities related to the inhibition of the caspase-11-mediated non-canonical activation of the inflammasome.

\section{Discussion}

It is estimated that as few as five infectious C. burnetii organisms are able to establish infection in healthy individuals exposed to the bacteria ${ }^{58}$. To achieve such a high infectious efficiency, C. burnetii inhibits a large repertoire of specific host cell responses, including those related to the innate immune activation and induction of host cell death. Although the inhibition of caspase-3-mediated apoptosis by C. burnetii has been reported previously ${ }^{29,31,32,35}$, an evaluation of inflammatory caspases and pyroptosis has never been conducted. Herein, we report that $C$. burnetii fails to induce caspase- 1 activation upon the infection of primary mouse macrophages. We found that the bacteria actively inhibit the caspase-11 activation and their functions including pore formation and the non-canonical activation of the NLRP3 inflammasome, but they do not inhibit the components of inflammasomes such as ASC, NLRP3 or caspase-1. By searching for the molecular mechanisms underlying this process, we identified a novel C. burnetii gene that encodes an

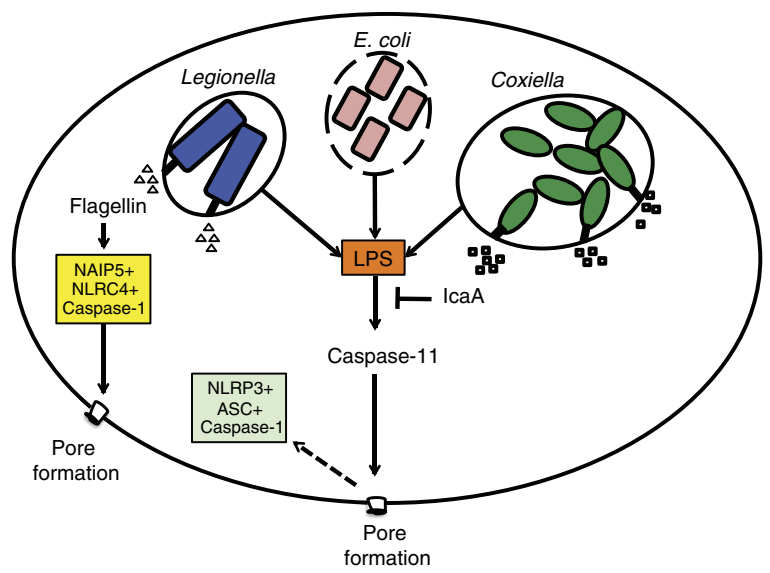

Figure 7 | Model for IcaA-mediated inhibition of non-canonical activation of the inflammasome. Published data demonstrate that caspase-11 senses the presence of cytosolic LPS from Gram-negative bacteria, including E. coli and L. pneumophila. Activation of caspase-11 in response to L. pneumophila requires the expression of Dot/lcm. The effector protein IcaA is translocated by the $C$. burnetii Dot/Icm and inhibits the caspase-11mediated pore formation and caspase-11-induced non-canonical activation of the NLRP3 inflammasome.

effector protein translocated by the Dot/Icm type IVB secretion system into host cell cytosol. We named this protein IcaA because it is functionally related to the inhibition of caspase activation. IcaA suppresses the caspase-11-mediated, non-canonical activation of caspase-1 in macrophages. Notably, we have no data to support a direct effect of IcaA in the inhibition of caspase11 activation. Most likely, IcaA is involved in the inhibition of 
certain cellular processes, which leads to caspase-11 activation (Fig. 7). It is also possible that IcaA operates in the vacuolar membranes by minimizing the release of bacterial molecules from the vacuole to the cytoplasm, thereby reducing the release of PAMPs that trigger cytosolic innate immune sensors. This hypothesis is consistent our observed ability to transfer the C. burnetii inhibitory activity to L. pneumophila in trans when IcaA was expressed in L. pneumophila. This was achieved because of the high homology of C. burnetii and L. pneumophila Dot $/ \mathrm{Icm}^{40}$. In fact, L. pneumophila has been extensively used as an effective surrogate host to investigate $C$. burnetii pathogenesis. Earlier studies demonstrated that the structural core components of the C. burnetii Dot/Icm can complement L. pneumophila mutants deficient in the respective homologous gene ${ }^{41,42}$. Further studies benefited from the high homology between the two Dot/ Icm and used L. pneumophila to actively translocate C. burnetii effector proteins ${ }^{44-50}$. This approach supported the identification of several effector proteins encoded by $C$. burnetii, including the protein encoded by gene CBU1823 (refs 44,45). However, only recently has the secretion of $C$. burnetii effectors via $C$. burnetii Dot/Icm been efficiently detected experimentally ${ }^{45}$. Notably, CBU1823 (IcaA) was one protein that has been used to demonstrate the functional activities of the C. burnetii Dot/Icm; therefore, IcaA is a bona fide effector secreted by the C. burnetii Dot/Icm system into the host cell cytoplasm ${ }^{55}$. These data demonstrate that IcaA is a bona fide substrate of the C. burnetii Dot/Icm, which can be translocated by the Legionella $\mathrm{Dot} / \mathrm{Icm}$ or by the Coxiella Dot/Icm ${ }^{44,45,55}$.

The genetic manipulation of $C$. burnetii has only recently been achieved $^{44,59}$. Therefore, little information is available regarding the role of specific C. burnetii effector proteins. It is believed that the bacterial effectors are determinants in the pathogenesis of this bacterium. A functional Dot/Icm system is required for C. burnetii growth in macrophages and in permissive cells, supporting an essential role of translocated effector proteins for bacterial survival ${ }^{44,59}$. By random transposon mutagenesis, several putative effectors have been shown to be important for bacterial replication ${ }^{48}$. Moreover, the deletion of CvpA, an effector that interacts with components of clathrin-coated endocytic vesicles, impairs the maturation of the parasitophorous vacuole and bacterial growth ${ }^{30}$. The gene-encoding IcaA studied herein was identified in the Nine Mile strain of $C$. burnetii, which is representative of acute disease isolates. Analysis of the genome of other important C. burnetii strains showed that this effector is conserved in the Dugway ${ }^{44}$, a strain isolated from rodents that is weakly pathogenic for guinea pigs. Notably, IcaA is encoded in truncated forms in the human endocarditis isolates $\mathrm{K}(\mathrm{Q} 154)$ and $\mathrm{G}(\mathrm{Q} 212)$ strains $^{44}$. Whether the IcaA is non-functional in $\mathrm{K}$ and $\mathrm{G}$ strains due to frameshift mutations and whether this reflects differences in the virulence and spreading of the bacteria to other tissues is a subject for future investigation. Notably, caspase-11-induced susceptibility to infection has been demonstrated in murine models of bacterial dissemination using Salmonella typhimurium ${ }^{60}$. In this context, it remains to be determined whether the IcaA-mediated inhibition of caspase-11 and pyroptosis regulates bacterial dissemination and accounts for the development of chronic versus acute $\mathrm{Q}$ fever. Another matter for further investigation is the effect of $i c a A$ in inflammasome activation within human macrophages. It was recently demonstrated that Nine Mile phase II can trigger the production of active IL- $1 \beta$ in primary human $\mathrm{AMs}^{61}$. Although we have demonstrated that the IcaA-mediated inhibition of caspase- 11 activation also occurs in primary mouse AMs, a direct comparison with primary human AMs will be important for the comprehensive assessment of the role of IcaA in the bacterial pathogenesis and host responses during $\mathrm{Q}$ fever. Nonetheless, the fact that primary human macrophages do trigger IL-1 $\beta$ production $^{61}$ supports our findings that C. burnetii does encode molecules responsible for the non-canonical activation of the inflammasome.

Regardless of the determination of the role of IcaA and caspase-11 in the outcome of C. burnetii infection in vivo, our study identifies IcaA as a novel bacterial substrate that participates in the inhibition of non-canonical inflammasome activation. To the best of our knowledge, this is the first bacterial effector that interferes with pyroptosis and the activation of the non-canonical inflammasome activation in macrophages ${ }^{19}$. By using epithelial cells, OspC3 was identified as a Shigella flexneri effector protein that functions as a direct inhibitor of human caspase-4 (ref. 62). This protein was shown to be important for bacterial pathogenesis using a guinea pig model of $S$. flexneri infection. However, it remains unclear whether OspC3 operates in macrophages and whether it interferes with the non-canonical activation of the inflammasome. Regardless of the effect of OspC3 in human epithelial cells, we propose that the C. burnetii effector protein IcaA interferes with the process involved in caspase-11 activation, consequently inhibiting non-canonical inflammasome activation in macrophages, a molecular platform that has recently emerged as major player in innate immune responses to Gram-negative bacteria ${ }^{12-18,53}$. C. burnetii does not inhibit caspase- 1 or other inflammasomes such as the NAIP5/NLRC4 or the canonical NLRP3. Because C. burnetii does not encode genes for flagellin expression, the bacteria may not have encountered selective pressure to develop molecules that target the activation of the NAIP5/NLRC4 inflammasome. In contrast, C. burnetii virulence is associated with the presence of the $\mathrm{O}$-antigen in its LPS containing a tetra-acylated lipid A with long fatty acid chains ${ }^{39}$. Because it has been recently reported that the non-canonical activation of the inflammasome proceeds through the intracellular recognition of cytosolic LPS ${ }^{16,17}$, it is possible that the C. burnetii LPS triggers this pathway, and the bacterium has evolutionary evolved effectors, such as IcaA, that facilitate the subversion of this important innate immune pathway. In support of this hypothesis, we found that the delivery of $C$. burnetii LPS into the macrophage cytoplasm triggers this pathway. Notably, tetra-acylated lipid A was reported to be inefficient for activating caspase-11 (ref. 16). It is possible that variations in the Coxiella lipid A occur according to the bacterial growth phase. Alternatively, it is possible that another bacterial molecule is responsible for triggering caspase-11-dependent pore formation. Nonetheless, the induction of pores in macrophages transfected with $C$. burnetii LPS indicates that $C$. burnetii can trigger a pathway that is inhibited by IcaA. Collectively, the identification of IcaA and the unravelling of its mechanistic functions effectively contribute to our understanding of the biology and pathogenesis of this highly infectious intracellular pathogen and provides novel molecular structures that can be used in the development of therapies for sepsis and chronic inflammatory diseases.

\section{Methods}

Bacterial strains. C. burnetii (strain Nine Mile RSA 493 phase II) were collected and purified from the infection of irradiated Vero cell monolayers in DMEM (Sigma-Aldrich) supplemented with $5 \%$ fetal bovine serum (FBS) at $37^{\circ} \mathrm{C}$ in $5 \% \mathrm{CO}_{2}$ (ref. 63). Legionella pneumophila. Wild-type (WT Lp) and isogenic flagellin-deficient mutants $\left(\right.$ flaA $\left.^{-}\right)$of L. pneumophila Lp02 (thymidine auxotrophic derivative from serogroup 1 strain Lp01) ${ }^{64}$ were used for co-infection assays. For the surrogate expression of C. burnetii effectors, WT Lp, flaA ${ }^{-}$and $\operatorname{dot} A^{-}$isogenic mutants from Lp01 (ref. 64) and $\mathrm{flaA}^{-}$isogenic mutants of Lp02 (ref. 64) and JR32 (ref. 65) strains were transformed as described below. L. pneumophila was cultivated at $37^{\circ} \mathrm{C}$ on MOPS-buffered charcoal-yeast extract (CYE) agar plates $(1 \%(\mathrm{w} / \mathrm{v})$ yeast extract, $1 \%(\mathrm{w} / \mathrm{v})$ MOPS, $3.3 \mathrm{mM}$ L-cysteine, $0.33 \mathrm{mM} \mathrm{Fe}\left(\mathrm{NO}_{3}\right)_{3}, 1.5 \%(\mathrm{w} / \mathrm{v})$ Bacto agar and $0.2 \%(\mathrm{w} / \mathrm{v})$ activated charcoal, $\mathrm{pH}$ 6.9) supplemented with $100 \mu \mathrm{g} \mathrm{ml}^{-1}$ thymidine, as appropriate. E. coli (DH5 $\alpha$ strain) was streaked at $37^{\circ} \mathrm{C}$ on Luria-Bertani (LB) agar plates without 
antibiotics for colony isolation and cultivated in $\mathrm{LB}$ broth at $37^{\circ} \mathrm{C}$ under rotation (200 r.p.m.)

Mice. C57BL/6, 129S6/SvEv and C57BL/6-derived $A s c^{-/-}$, Casp11 $11^{-/-}$,

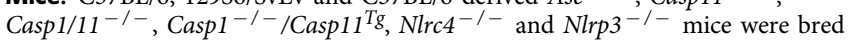
and maintained under specific pathogen-free conditions in the Animal Facilities of the Medical School Ribeirão Preto (FMRP-USP). Casp $1^{-/} / \operatorname{Casp}_{1} 1^{\mathrm{Tg}}$ mice are Casp $1 / 11^{-/}$mice expressing a transgene encoding a functional copy of the caspase-11 allele, as described previously ${ }^{53}$. Male or female mice with $8-12$ weeks old were used. All mouse experiments were conducted according to the guidelines of the institutional committee for animal care at the Comissão de Ética em Experimentação Animal da Faculdade de Medicina de Ribeirão Preto, FMRP-USP.

\section{Plasmid construction and expression of Dot/lcm effectors of $\boldsymbol{C}$. burnetii in} L. pneumophila. The $\mathrm{pSN} 85 / \mathrm{Cm}^{+}$plasmid with a N-terminal Flag epitope tag was used to clone Dot/Icm effectors from C. burnetii (strain Nine Mile RSA 493 phase II). Full-length genes of effectors annotated as CBU were subcloned from pEC33 constructs $^{44}$ through BamHI and PstI sites. Full-length genes of effectors annotated as Ank were amplified from purified genomic DNA ${ }^{44}$ by PCR with sequence-specific primers containing Bam $\mathrm{HI}$ and SphI sites or XbaI and SphI in the case of AnkB (all primers used in this study are provided in Supplementary Table 1). For AnkJ, genomic DNA from the Dugway isolate was amplified by PCR with sequence-specific primers containing BamHI and SphI sites. Following heatshock transformation and amplification in E. coli (DH5 $\alpha$ strain), the constructs were purified with a NucleoSpin plasmid kit (Macherey-Nagel) and subsequently used to transform L. pneumophila by electroporation. Transformed L. pneumophila clones were selected and expanded in CYE plates with $10 \mu \mathrm{g} \mathrm{ml}^{-1}$

chloramphenicol. Effector expression was induced with the addition of $1 \mathrm{mM}$ isopropyl $\beta$-D-1-thiogalactopyranoside (IPTG, Invitrogen) to plates and confirmed by immunoblot using an anti-Flag antibody (Sigma-Aldrich).

Construction of icaA $\boldsymbol{A}^{-}$mutants of $\boldsymbol{C}$. burnetii. A PCR product consisting of CBU1823 and $2 \mathrm{~kb}$ of DNA flanking either end was amplified from C. burnetii (Nine Mile RSA 493 phase II) genomic DNA by using PfuUltra II Fusion HotStart DNA polymerase (Agilent Technologies). The product was ligated into the BamHI and SalI sites of pJC-CAT. The resulting construct, pJC-1823FL, was then used as a template in a PCR. The resulting PCR product was then gel purified and digested with NotI-HF (New England Biolabs) and ligated with the kanamycin resistance gene amplified from pJB-Kan. The ligation mix was then transformed into $\mathrm{DH} 5 \alpha$. The resulting construct, pJC1823KO, in which the CBU1823 gene was replaced with a kanamycin resistance gene, was then used in the gene knockout experiment ${ }^{66}$. CBU1823 knockout was confirmed by Southern hybridization using probes directly binding to CBU1823 and the kanamycin resistance cassette.

Bone marrow-derived and alveolar macrophages. AMs were obtained by bronchoalveolar lavage with warm PBS containing 5 mM EDTA. AMs were washed twice with PBS, seeded on tissue culture plates with RPMI 1640 media $10 \%$ FBS, $2 \mathrm{mM}$ L-glutamine and cultivated at $36^{\circ} \mathrm{C}, 5 \% \mathrm{CO}_{2}$. C. burnetii replication in AMs was assessed by quantitative PCR, as described previously ${ }^{67}$. Bone marrow cells were collected from femurs and differentiated with RPMI 1640 (Sigma-Aldrich), $20 \%$ FBS (Invitrogen), 30\% L-929 cell-conditioned media (LCCM), 2 mM L-glutamine (Sigma-Aldrich) and $100 \mathrm{U} \mathrm{ml}^{-1}$ penicillin-streptomycin (SigmaAldrich) cultivated at $36^{\circ} \mathrm{C}, 5 \% \mathrm{CO}_{2}$. BMDMs were collected and seeded on tissue culture plates and kept in RPMI 1640 media 10\% FBS, 5\% LCCM and 2 mM L-glutamine ${ }^{68}$.

Construction of retroviral expression vectors and transduction of BMDMs. Full-length genomic C57BL/6 Casp11 was amplified by PCR with sequence-specific primers containing NotI and XhoI sites for cloning into pCDNA3.1/Hygro ${ }^{+}$vector (Invitrogen). Casp11 was subsequently subcloned into a pMSCV2.0 murine-specific retroviral vector after the digestion of the purified pCDNA3 construct with Not I and SalI. pCL vector system ${ }^{69}$ for packaging retroviruses was co-transfected with pMSCV-Casp11-IRES-GFP using polyethylenimine in monolayers of Peak cells (ATCC) maintained in RPMI 1640 with $10 \% \mathrm{FBS}$ at $36^{\circ} \mathrm{C}$ and $5 \% \mathrm{CO}_{2}$. BMDMs in day 3 of differentiation were collected, spun down and resuspended in retrovirus-containing peak cell supernatant supplemented with RPMI 1640 containing 20\% FBS and 25\% LCCM. Cell transduction was confirmed by fluorescence microscopy. BMDMs were then collected, seeded on tissue culture plates one day before infection and kept in RPMI 1640 media 10\% FBS and 5\% LCCM.

Infection conditions. C. burnetii from frozen stocks was thawed at $37^{\circ} \mathrm{C}$ L. pneumophila single colonies were seeded and grown for 2 days in CYE agar plates, collected, diluted in distilled water and quantified by measuring optical density (OD) at $600 \mathrm{~nm}$. L. pneumophila mutants expressing C. burnetii effectors were infected in the presence of $1 \mathrm{mM}$ IPTG. E. coli single colonies were grown in $\mathrm{LB}$ broth for $8 \mathrm{~h}$ and quantified by measuring OD at $600 \mathrm{~nm}$. E. coli-infected macrophages were washed twice with warm PBS one hour after infection, and the media were replaced with media containing gentamicin (Sigma-Aldrich,

$50 \mu \mathrm{g} \mathrm{ml}^{-1}$ ). When necessary, the plates were centrifuged for $15 \mathrm{~min}$ at $300 \mathrm{~g}$ to ensure the comparable adhesion of the bacteria to the cells.

Extraction of $\boldsymbol{C}$. burnetii LPS. LPS was extracted using a modified hot phenol method, as described previously ${ }^{70}$. Briefly, $10^{6}$ C. burnetii were suspended in $1 \mathrm{ml}$ of $50 \%$ phenol ( $\mathrm{pH} 8.8$ ), boiled for $10 \mathrm{~min}$, incubated for $5 \mathrm{~min}$ on ice and then centrifuged at $14,000 \mathrm{~g}$ for $5 \mathrm{~min}$. The aqueous phase was collected, and the extraction was repeated on the pellet. The aqueous phases from both extractions were pooled and vacuum dried for $17 \mathrm{~h}$. The pellet was dissolved in $100 \mu \mathrm{l}$ of ultra-pure distilled $\mathrm{H}_{2} \mathrm{O}$. The LPS content after extraction was estimated by a phenol-sulfuric acid method of carbohydrate quantification. Approximately, $1 \mu \mathrm{g} \mathrm{ml}^{-1}$ of $C$. burnetii LPS were used to transfect BMDMs using DOTAP (Roche) according to the manufacturer's instructions.

Determination of $\boldsymbol{L}$. pneumophila number per Legionella-containing vacuole (LCV). BMDMs were cultivated in coverslips and fixed with 4\% PFA after infection. The coverslips were immunostained with rabbit anti-Legionella $(1: 2,000)$ and goat anti-rabbit IgG Alexa Fluor 488 (Molecular Probes), mounted in glass slides with ProLong Gold containing DAPI (Molecular Probes). The coverslips were analysed with a fluorescence microscope (Leica, Germany), and the number of bacteria per LCV was estimated visually.

Immunoblot analysis. BMDMs were lysed with a RIPA buffer $(10 \mathrm{mM}$ Tris- $\mathrm{HCl}$ (pH 7.4), 1 mM EDTA, $150 \mathrm{mM} \mathrm{NaCl}, 1 \%$ Nonidet P-40, $1 \%$ (w/v) sodium deoxycholate and $0.1 \%(\mathrm{w} / \mathrm{v})$ SDS) supplemented with a protease inhibitor cocktail. Precleared lysates and supernatants were boiled with Laemmli buffer, resolved by SDS-polyacrylamide gel electrophoresis and transferred to a $0.22-\mu \mathrm{m}$ nitrocellulose membrane (GE Healthcare). Rat anti-caspase-1 p20 monoclonal antibody clone 4B4 (Genentech, 1:500), mouse anti-caspase-1 p10 (Santa Cruz Biotechnology, 1:250), goat anti-IL-1 $\beta$ p17 subunit (Sigma-Aldrich, 1:250), rat anti-caspase-11 p10 (Abcam, 1:500) and rabbit anti-beta actin (Sigma-Aldrich, 1:5,000) were used for antigen detection. To detect the expression of Flag-fused effectors by L. pneumophila, bacterial heavy patches streaked from CYE plates after 2 days of growth in the presence of $1 \mathrm{mM}$ IPTG were boiled in Laemmli buffer, immunoblotting was carried out as described above, and anti-Flag M2 (Sigma-Aldrich, 1:5,000) antibody was used for antigen detection.

Reagents for cell stimulation and cytokine determination. Ultra-pure LPS (E. coli K12) and poli I:C were from Invivogen. Tumour-necrosis factor- $\alpha$ was from eBioscience. ATP, nigericin and CTB were from Sigma-Aldrich. Cytokines from supernatants of the BMDM culture were detected by ELISA (OptEIA, BD Biosciences), according to the manufacturer's instructions.

Membrane pore formation assay. Pore formation in BMDMs and AMs was quantified by the permeability to ethidium bromide in damaged cells ${ }^{57}$. Images were acquired with a fluorescence microscope (Leica, Germany), five fields per coverslip. Pore formation was quantified using ImageJ software (NIH). For the kinetics of pore formation, the uptake of PI in infected cells was evaluated ${ }^{13}$. BMDMs were seeded in a black, clear-bottom 96-well plate. Before infection or stimulation, BMDMs were washed with warm PBS, and the media were replenished with RPMI $10 \%$ without phenol red, $0.038 \mathrm{~g} \mathrm{ml}^{-1} \mathrm{NaHCO}_{3}$, $6 \mu \mathrm{g} \mathrm{ml}^{-1}$ PI. Infected BMDMs were kept at $37^{\circ} \mathrm{C}$, and PI was excited at $538 \mathrm{~nm}$. The fluorescence emission was read at $617 \mathrm{~nm}$ at every $5 \mathrm{~min}$ using a plate fluorometer (FlexStation 3, Molecular Devices).

FLICA staining and flow cytometry analysis. BMDMs were stained with a caspase-1 FLICA probe (FAM-YVAD-FMK), as recommended by the manufacturer. HEK293 reporter cells were likewise stained with a pan-caspase FLICA probe (carboxyfluorescein-VAD-fluoromethylketone, FAM-VAD-FMK; ImmunoChemistry Technologies). Data from 30,000 events were acquired on a FACSCanto II (BD Biosciences) and were analysed with FlowJo software (Tree Star).

Active caspase-11 pull-down assay. The media of primed BMDMs were replenished with fresh media containing $20 \mu \mathrm{M}$ biotin-VAD-FMK (Enzo) $15 \mathrm{~min}$ before infection. Infected BMDMs were lysed in RIPA buffer $(10 \mathrm{mM}$ Tris- $\mathrm{HCl}$ ( $\mathrm{pH}$ 7.4), $1 \mathrm{mM}$ EDTA, $150 \mathrm{mM} \mathrm{NaCl}, 1 \%$ Nonidet P-40, 1\% (w/v) sodium deoxycholate and $0.1 \%(\mathrm{w} / \mathrm{v})$ SDS) supplemented with a protease inhibitor cocktail (Roche). Cleared lysates were equalized according to total protein content, incubated overnight with streptavidin-agarose beads (Novex) and thoroughly rinsed with RIPA buffer. Bound proteins were eluted by re-suspension in Laemmli sample buffer, boiled for $5 \mathrm{~min}$ and separated by SDS-polyacrylamide gel electrophoresis. 
Statistical analysis. The data were plotted and analysed using GraphPad Prism 5.0 software. The statistical significance was calculated using Student's $t$-test or analysis of variance (ANOVA). Differences were considered statistically significant when the $P$ value was $<0.05$.

\section{References}

1. Janeway, Jr C. A. Approaching the asymptote? Evolution and revolution in immunology. Cold Spring Harb. Symp. Quant. Biol. 54(Pt 1): 1-13 (1989).

2. Mariathasan, S. \& Monack, D. M. Inflammasome adaptors and sensors: intracellular regulators of infection and inflammation. Nat. Rev. Immunol. 7, 31-40 (2007).

3. Martinon, F., Burns, K. \& Tschopp, J. The inflammasome: a molecular platform triggering activation of inflammatory caspases and processing of proIL-beta. Mol. Cell 10, 417-426 (2002).

4. Miao, E. A. et al. Cytoplasmic flagellin activates caspase-1 and secretion of interleukin 1beta via Ipaf. Nat. Immunol. 7, 569-575 (2006).

5. Miao, E. A., Ernst, R. K., Dors, M., Mao, D. P. \& Aderem, A. Pseudomonas aeruginosa activates caspase 1 through Ipaf. Proc. Natl Acad. Sci. USA 105, 2562-2567 (2008).

6. Miao, E. A. et al. Innate immune detection of the type III secretion apparatus through the NLRC4 inflammasome. Proc. Natl Acad. Sci. USA 107, 3076-3080 (2010).

7. Kofoed, E. M. \& Vance, R. E. Innate immune recognition of bacterial ligands by NAIPs determines inflammasome specificity. Nature 477, 592-595 (2011).

8. Franchi, L. et al. Critical role for Ipaf in Pseudomonas aeruginosa-induced caspase-1 activation. Eur. J. Immunol. 37, 3030-3039 (2007).

9. Amer, A. et al. Regulation of Legionella phagosome maturation and infection through flagellin and host Ipaf. J. Biol. Chem. 281, 35217-35223 (2006).

10. Ren, T., Zamboni, D. S., Roy, C. R., Dietrich, W. F. \& Vance, R. E. Flagellin-deficient Legionella mutants evade caspase-1- and Naip5-mediated macrophage immunity. PLoS Pathogens 2, e18 (2006).

11. Zamboni, D. S. et al. The Bircle cytosolic pattern-recognition receptor contributes to the detection and control of Legionella pneumophila infection. Nat. Immunol. 7, 318-325 (2006).

12. Aachoui, Y. et al. Caspase-11 protects against bacteria that escape the vacuole. Science 339, 975-978 (2013).

13. Case, C. L. et al. Caspase-11 stimulates rapid flagellin-independent pyroptosis in response to Legionella pneumophila. Proc. Nl Acad. Sci. USA 110, 1851-1856 (2013).

14. Casson, C. N. et al. Caspase-11 activation in response to bacterial secretion systems that access the host cytosol. PLoS Pathogens 9, e1003400 (2013).

15. Gurung, P. et al. Toll or interleukin-1 receptor (TIR) domain-containing adaptor inducing interferon-beta (TRIF)-mediated caspase-11 protease production integrates Toll-like receptor 4 (TLR4) protein- and Nlrp3 inflammasome-mediated host defense against enteropathogens. J. Biol. Chem. 287, 34474-34483 (2012).

16. Hagar, J. A., Powell, D. A., Aachoui, Y., Ernst, R. K. \& Miao, E. A. Cytoplasmic LPS activates caspase-11: implications in TLR4-independent endotoxic shock. Science 341, 1250-1253 (2013).

17. Kayagaki, N. et al. Noncanonical inflammasome activation by intracellular LPS independent of TLR4. Science 341, 1246-1249 (2013).

18. Rathinam, V. A. et al. TRIF licenses caspase-11-dependent NLRP3 inflammasome activation by gram-negative bacteria. Cell 150, 606-619 (2012).

19. Cunha, L. D. \& Zamboni, D. S. Subversion of inflammasome activation and pyroptosis by pathogenic bacteria. Front. Cell. Infect. Microbiol. 3, 76 (2013).

20. Nagai, H. \& Roy, C. R. Show me the substrates: modulation of host cell function by type IV secretion systems. Cell. Microbiol. 5, 373-383 (2003).

21. Galan, J. E. \& Wolf-Watz, H. Protein delivery into eukaryotic cells by type III secretion machines. Nature 444, 567-573 (2006).

22. Baxt, L. A., Garza-Mayers, A. C. \& Goldberg, M. B. Bacterial subversion of host innate immune pathways. Science 340, 697-701 (2013).

23. Jorgensen, I. et al. The Chlamydia protease CPAF regulates host and bacterial proteins to maintain pathogen vacuole integrity and promote virulence. Cell Host Microbe 10, 21-32 (2011).

24. Danelishvili, L., Everman, J. L., McNamara, M. J. \& Bermudez, L. E. Inhibition of the plasma-membrane-associated serine protease cathepsin $\mathrm{G}$ by Mycobacterium tuberculosis Rv3364c suppresses caspase-1 and pyroptosis in macrophages. Front. Microbiol. 2, 281 (2011).

25. Shah, S. et al. Cutting edge: Mycobacterium tuberculosis but not nonvirulent mycobacteria inhibits IFN-beta and AIM2 inflammasome-dependent IL-1beta production via its ESX-1 secretion system. J. Immunol. 191, 3514-3518 (2013).

26. Raoult, D., Marrie, T. \& Mege, J. Natural history and pathophysiology of Q fever. Lancet Infect. Dis. 5, 219-226 (2005).

27. van Schaik, E. J., Chen, C., Mertens, K., Weber, M. M. \& Samuel, J. E. Molecular pathogenesis of the obligate intracellular bacterium Coxiella burnetii. Nat. Rev. Microbiol. 11, 561-573 (2013).
28. Campoy, E. M., Zoppino, F. C. \& Colombo, M. I. The early secretory pathway contributes to the growth of the Coxiella-replicative niche. Infect. Immun. 79, 402-413 (2011).

29. Klingenbeck, L., Eckart, R. A., Berens, C. \& Luhrmann, A. The Coxiella burnetii type IV secretion system substrate CaeB inhibits intrinsic apoptosis at the mitochondrial level. Cell. Microbiol. 15, 675-687 (2012).

30. Larson, C. L., Beare, P. A., Howe, D. \& Heinzen, R. A. Coxiella burnetii effector protein subverts clathrin-mediated vesicular trafficking for pathogen vacuole biogenesis. Proc. Natl Acad. Sci. USA 110, E4770-E4779 (2013).

31. Luhrmann, A., Nogueira, C. V., Carey, K. L. \& Roy, C. R. Inhibition of pathogen-induced apoptosis by a Coxiella burnetii type IV effector protein. Proc. Natl Acad. Sci. USA 107, 18997-19001 (2010).

32. Luhrmann, A. \& Roy, C. R. Coxiella burnetii inhibits activation of host cell apoptosis through a mechanism that involves preventing cytochrome $\mathrm{c}$ release from mitochondria. Infect. Immun. 75, 5282-5289 (2007).

33. McDonough, J. A. et al. Host pathways important for Coxiella burnetii infection revealed by genome-wide RNA interference screening. MBio 4, e00606-e00612 (2013).

34. Voth, D. E. \& Heinzen, R. A. Sustained activation of Akt and Erk1/2 is required for Coxiella burnetii antiapoptotic activity. Infect. Immun. 77, 205-213 (2009).

35. Voth, D. E., Howe, D. \& Heinzen, R. A. Coxiella burnetii inhibits apoptosis in human THP-1 cells and monkey primary alveolar macrophages. Infect. Immun. 75, 4263-4271 (2007).

36. Vazquez, C. L. \& Colombo, M. I. Coxiella burnetii modulates Beclin 1 and $\mathrm{Bcl}-2$, preventing host cell apoptosis to generate a persistent bacterial infection. Cell Death Differ. 17, 421-438 (2010).

37. Shannon, J. G., Howe, D. \& Heinzen, R. A. Virulent Coxiella burnetii does not activate human dendritic cells: role of lipopolysaccharide as a shielding molecule. Proc. Natl Acad. Sci. USA 102, 8722-8727 (2005).

38. Zamboni, D. S. Genetic control of natural resistance of mouse macrophages to Coxiella burnetii infection in vitro: macrophages from restrictive strains control parasitophorous vacuole maturation. Infect. Immun. 72, 2395-2399 (2004).

39. Zamboni, D. S. et al. Stimulation of toll-like receptor 2 by Coxiella burnetii is required for macrophage production of pro-inflammatory cytokines and resistance to infection. J. Biol. Chem. 279, 54405-54415 (2004).

40. Seshadri, R. et al. Complete genome sequence of the Q-fever pathogen Coxiella burnetii. Proc. Natl Acad. Sci. USA 100, 5455-5460 (2003).

41. Zamboni, D. S., McGrath, S., Rabinovitch, M. \& Roy, C. R. Coxiella burnetii express type IV secretion system proteins that function similarly to components of the Legionella pneumophila Dot/Icm system. Mol. Microbiol. 49, 965-976 (2003).

42. Zusman, T., Yerushalmi, G. \& Segal, G. Functional similarities between the icm/ dot pathogenesis systems of Coxiella burnetii and Legionella pneumophila. Infect. Immun. 71, 3714-3723 (2003).

43. Beare, P. A. et al. Comparative genomics reveal extensive transposon-mediated genomic plasticity and diversity among potential effector proteins within the genus Coxiella. Infect. Immun. 77, 642-656 (2009).

44. Carey, K. L., Newton, H. J., Luhrmann, A. \& Roy, C. R. The Coxiella burnetii Dot/Icm system delivers a unique repertoire of type IV effectors into host cells and is required for intracellular replication. PLoS Pathogens 7, e1002056 (2011).

45. Chen, C. et al. Large-scale identification and translocation of type IV secretion substrates by Coxiella burnetii. Proc. Natl Acad. Sci. USA 107, 21755-21760 (2010).

46. Voth, D. E. et al. The Coxiella burnetii cryptic plasmid is enriched in genes encoding type IV secretion system substrates. J. Bacteriol. 193, 1493-1503 (2011).

47. Voth, D. E. et al. The Coxiella burnetii ankyrin repeat domain-containing protein family is heterogeneous, with C-terminal truncations that influence Dot/Icm-mediated secretion. J. Bacteriol. 191, 4232-4242 (2009).

48. Weber, M. M. et al. Identification of Coxiella burnetii type IV secretion substrates required for intracellular replication and Coxiella-containing vacuole formation. J. Bacteriol. 195, 3914-3924 (2013).

49. Lifshitz, Z. et al. Computational modeling and experimental validation of the Legionella and Coxiella virulence-related type-IVB secretion signal. Proc. Natl Acad. Sci. USA 110, E707-E715 (2013).

50. Pan, X., Luhrmann, A., Satoh, A., Laskowski-Arce, M. A. \& Roy, C. R. Ankyrin repeat proteins comprise a diverse family of bacterial type IV effectors. Science 320, 1651-1654 (2008).

51. Sauer, J. D. et al. Specificity of Legionella pneumophila and Coxiella burnetii vacuoles and versatility of Legionella pneumophila revealed by coinfection. Infect. Immun. 73, 4494-4504 (2005).

52. Case, C. L., Shin, S. \& Roy, C. R. Asc and Ipaf Inflammasomes direct distinct pathways for caspase-1 activation in response to Legionella pneumophila. Infect. Immun. 77, 1981-1991 (2009).

53. Kayagaki, N. et al. Non-canonical inflammasome activation targets caspase-11. Nature 479, 117-121 (2011). 
54. Cerqueira, D. M., Pereira, M. S., Silva, A. L., Cunha, L. D. \& Zamboni, D. S. Caspase-1 but not caspase-11 is required for NLRC4-mediated pyroptosis and restriction of infection by flagellated Legionella species in mouse macrophages and in vivo. J. Immunol. 195, 2303-2311 (2015).

55. Newton, H. J., McDonough, J. A. \& Roy, C. R. Effector protein translocation by the Coxiella burnetii Dot/Icm type IV secretion system requires endocytic maturation of the pathogen-occupied vacuole. PLOS ONE 8, e54566 (2013).

56. Molofsky, A. B. et al. Cytosolic recognition of flagellin by mouse macrophages restricts Legionella pneumophila infection. J. Exp. Med. 203, 1093-1104 (2006).

57. Silveira, T. N. \& Zamboni, D. S. Pore formation triggered by Legionella spp. is an Nlrc4 inflammasome-dependent host cell response that precedes pyroptosis. Infect. Immun. 78, 1403-1413 (2010).

58. Brooke, R. J., Kretzschmar, M. E., Mutters, N. T. \& Teunis, P. F. Human dose response relation for airborne exposure to Coxiella burnetii. BMC Infect. Dis. 13, 488 (2013).

59. Beare, P. A. et al. Dot/Icm type IVB secretion system requirements for Coxiella burnetii growth in human macrophages. MBio 2, e00175-e00211 (2011).

60. Broz, P. et al. Caspase-11 increases susceptibility to Salmonella infection in the absence of caspase-1. Nature 490, 288-291 (2012).

61. Graham, J. G. et al. Virulent Coxiella burnetii pathotypes productively infect primary human alveolar macrophages. Cell. Microbiol. 15, 1012-1025 (2013).

62. Kobayashi, T. et al. The Shigella OspC3 effector inhibits caspase-4, antagonizes inflammatory cell death, and promotes epithelial infection. Cell Host Microbe 13, 570-583 (2013).

63. Zamboni, D. S., Mortara, R. A. \& Rabinovitch, M. Infection of Vero cells with Coxiella burnetii phase II: relative intracellular bacterial load and distribution estimated by confocal laser scanning microscopy and morphometry. $J$. Microbiol. Methods 43, 223-232 (2001).

64. Berger, K. H. \& Isberg, R. R. Two distinct defects in intracellular growth complemented by a single genetic locus in Legionella pneumophila. Mol. Microbiol. 7, 7-19 (1993).

65. Sadosky, A. B., Wiater, L. A. \& Shuman, H. A. Identification of Legionella pneumophila genes required for growth within and killing of human macrophages. Infect. Immun. 61, 5361-5373 (1993).

66. Beare, P. A. \& Heinzen, R. A. Gene inactivation in Coxiella burnetii. Methods Mol. Biol. 1197, 329-345 (2014).

67. Brennan, R. E. \& Samuel, J. E. Evaluation of Coxiella burnetii antibiotic susceptibilities by real-time PCR assay. J. Clin. Microbiol. 41, 1869-1874 (2003).

68. Marim, F. M., Silveira, T. N., Lima, Jr D. S. \& Zamboni, D. S. A method for generation of bone marrow-derived macrophages from cryopreserved mouse bone marrow cells. PLoS ONE 5, e15263 (2010).

69. Naviaux, R. K., Costanzi, E., Haas, M. \& Verma, I. M. The pCL vector system: rapid production of helper-free, high-titer, recombinant retroviruses. J. Virol. 70, 5701-5705 (1996).
70. Beare, P. A., Howe, D., Cockrell, D. C. \& Heinzen, R. A. Efficient method of cloning the obligate intracellular bacterium Coxiella burnetii. Appl. Environ. Microbiol. 73, 4048-4054 (2007).

\section{Acknowledgements}

This paper is dedicated to Dr Michel Rabinovitch, whose passion for biology and scientific investigation co-infects his students forever. We thank Maira Nakamura for her technical assistance, to Richard Flavell (Yale, USA) for providing us with Nlrc4 $4^{-1}$ and Casp $1 / 11^{-/-}$mice and Vishva Dixit (Genentech, USA) for providing us with Nlrp $3^{-I-}$, Casp $^{-/-} /$Casp $11^{\mathrm{Tg}}$ and Casp $11^{-/-}$and the antibody to caspase-1 p20. This work was supported by grants from Fundação de Amparo à Pesquisa do Estado de São Paulo (FAPESP, grants 2013/08216-2, 2014/50268-2 and 2014/04684-4), Conselho Nacional do Desenvolvimento Cientifico e Tecnológico (CNPq and INCTV/CNPq), Coordenação de Aperfeiçoamento de Pessoal de Nível Superior (CAPES), Núcleo de Apoio à Pesquisa em Doenças Inflamatórias (NAPDIN, grant 11.1.21625.01.0) and the PEW Program in Biomedical Sciences. L.D.C. and J.M.R. are supported by fellowships from FAPESP. L.M.M. and T.D.F. are supported by fellowships from CAPES. H.J.N. is a visiting professor from Science Without Borders Program (CNPq, grant 401577/2014-7). D.S.Z. is a research fellow from $\mathrm{CNPq}$, Brazil.

\section{Author contributions}

L.D.C., T.D.F., H.J.N., C.R.R. and D.S.Z. designed the experiments; L.D.C., J.M.R., T.D.F., L.M.M., C.A.K., J.H.M. and H.J.N. performed the experiments; L.D.C., J.M.R., T.D.F. and D.S.Z. processed and analysed the data; L.D.C. and D.S.Z. wrote the manuscript; and all authors commented on the manuscript.

\section{Additional information}

Supplementary Information accompanies this paper at http://www.nature.com/ naturecommunications

Competing financial interests: The authors declare no competing financial interests.

Reprints and permission information is available online at http://npg.nature.com/ reprintsandpermissions/

How to cite this article: Cunha, L. D. et al. Inhibition of inflammasome activation by Coxiella burnetii type IV secretion system effector IcaA. Nat. Commun. 6:10205 doi: 10.1038/ncomms10205 (2015).

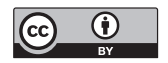

This work is licensed under a Creative Commons Attribution 4.0 International License. The images or other third party material in this article are included in the article's Creative Commons license, unless indicated otherwise in the credit line; if the material is not included under the Creative Commons license, users will need to obtain permission from the license holder to reproduce the material. To view a copy of this license, visit http://creativecommons.org/licenses/by/4.0/ 